University of Nebraska - Lincoln

DigitalCommons@University of Nebraska - Lincoln

Public Health Resources

Public Health Resources

2007

\title{
Comparative Genetic Analysis of Genomic DNA Sequences of Two Human Isolates of Tanapox virus
}

\author{
Steven H. Nazarian \\ Biotherapeutics Research Group, Robarts Research Institute, and Department of Microbiology and \\ Immunology, University of Western Ontario, London, Ontario N6G 2V4, Canada \\ John W. Barrett \\ Biotherapeutics Research Group, Robarts Research Institute, and Department of Microbiology and \\ Immunology, University of Western Ontario, London, Ontario N6G 2V4, Canada
}

\begin{abstract}
A. Michael Frace
Biotechnology Core Facility Branch, Division of Scientific Resources, National Center for Preparedness, Detection, and Control of Infectious Diseases, Centers for Disease Control and Prevention, Atlanta, GA 30329, USA

Melissa Olsen-Rasmussen

Biotechnology Core Facility Branch, Division of Scientific Resources, National Center for Preparedness, Detection, and Control of Infectious Diseases, Centers for Disease Control and Prevention, Atlanta, GA 30329, USA

Marina Khristova

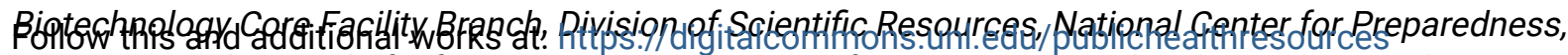
Dotection, and Control of Infectious Diseases, Centers for Disease Control and Prevention, Atlanta, GA 3Parturf Ahe Public Health Commons
\end{abstract}

See next page for additional authors

Nazarian, Steven H.; Barrett, John W.; Frace, A. Michael; Olsen-Rasmussen, Melissa; Khristova, Marina; Shaban, Mae; Neering, Sarah; Li, Yu; Damon, Inger K.; Esposito, Joseph J.; Essani, Karim; and McFadden, Grant, "Comparative Genetic Analysis of Genomic DNA Sequences of Two Human Isolates of Tanapox virus" (2007). Public Health Resources. 61.

https://digitalcommons.unl.edu/publichealthresources/61

This Article is brought to you for free and open access by the Public Health Resources at DigitalCommons@University of Nebraska - Lincoln. It has been accepted for inclusion in Public Health Resources by an authorized administrator of DigitalCommons@University of Nebraska - Lincoln. 


\section{Authors}

Steven H. Nazarian, John W. Barrett, A. Michael Frace, Melissa Olsen-Rasmussen, Marina Khristova, Mae Shaban, Sarah Neering, Yu Li, Inger K. Damon, Joseph J. Esposito, Karim Essani, and Grant McFadden 


\title{
Comparative genetic analysis of genomic DNA sequences of two human isolates of Tanapox virus ${ }^{\text {is }}$
}

\author{
Steven H. Nazarian ${ }^{a}$, John W. Barrett ${ }^{\mathrm{a}}$, A. Michael Frace ${ }^{\mathrm{b}}$, Melissa Olsen-Rasmussen ${ }^{\mathrm{b}}$, \\ Marina Khristova ${ }^{\mathrm{b}}$, Mae Shaban ${ }^{\mathrm{a}}$, Sarah Neering ${ }^{\mathrm{d}}$, Yu Li ${ }^{\mathrm{c}}$, Inger K. Damon ${ }^{\mathrm{c}}$, \\ Joseph J. Esposito ${ }^{\mathrm{b}}$, Karim Essani ${ }^{\mathrm{d}}$, Grant McFadden ${ }^{\mathrm{a}, *}$ \\ ${ }^{a}$ Biotherapeutics Research Group, Robarts Research Institute, and Department of Microbiology and Immunology, \\ University of Western Ontario, London, Ontario N6G 2V4, Canada \\ ${ }^{\mathrm{b}}$ Biotechnology Core Facility Branch, Division of Scientific Resources, National Center for Preparedness, Detection, \\ and Control of Infectious Diseases, Centers for Disease Control and Prevention, Atlanta, GA 30329, USA \\ ${ }^{\mathrm{c}}$ Poxvirus and Rabiesvirus Branch, Division of Viral and Rickettsial Diseases, National Center for Zoonotic, Vector-Borne, \\ and Enteric Diseases, Centers for Disease Control and Prevention, Atlanta, GA 30329, USA \\ ${ }^{\mathrm{d}}$ Laboratory of Virology, Department of Biological Science, Western Michigan University, Kalamazoo, MI 49008, USA \\ Received 10 March 2007; received in revised form 1 May 2007; accepted 1 May 2007 \\ Available online 14 June 2007
}

\begin{abstract}
Members of the genus Yatapoxvirus, which include Tanapox virus (TPV) and Yaba monkey tumor virus, infect primates including humans. Two strains of TPV isolated 50 years apart from patients infected from the equatorial region of Africa have been sequenced. The original isolate from a human case in the Tana River Valley, Kenya, in 1957 (TPV-Kenya) and an isolate from an infected traveler in the Republic of Congo in 2004 (TPV-RoC). Although isolated 50 years apart the genomes were highly conserved. The genomes differed at only 35 of 144,565 nucleotide positions (99.98\% identical). We predict that TPV-RoC encodes 155 ORFs, however a single transversion (at nucleotide 10241) in TPV-Kenya resulted in the coding capacity for two predicted ORFs $(11.1 \mathrm{~L}$ and $11.2 \mathrm{~L})$ in comparison to a single ORF (11L) in TPV-RoC. The genomes of TPV are $\mathrm{A}+\mathrm{T}$ rich $(73 \%)$ and $96 \%$ of the sequence encodes predicted ORFs. Comparative genomic analysis identified several features shared with other chordopoxviruses. A conserved sequence within the terminal inverted repeat region that is also present in the other members of the Yatapoxviruses as well as members of the Capripoxviruses, Swinepox virus and an unclassified Deerpox virus suggests the existence of a conserved near-terminal sequence secondary structure. Two previously unidentified gene families were annotated that are represented by ORF TPV28L, which matched homologues in certain other chordopoxviruses, and TPV42.5L, which is highly conserved among currently reported chordopoxvirus sequences.
\end{abstract}

Keywords: Tanapox; Yatapoxvirus; Poxvirus; Comparative genomics

\section{Introduction}

Poxviruses constitute two sub-families, Chordopoxvirinae and Entomopoxvirinae, which infect a wide range of ver-

\footnotetext{
is Disclaimer: The findings and conclusions in this report are those of the authors and do not necessarily represent the views of the funding agencies. Use of trade names or commercial sources is for identification only and does not imply endorsement by the funding agencies.

* Corresponding author. Present address: University of Florida, 1600 SW Archer Road, ARB Room R4-295, P.O. Box 100332, Gainesville, FL 32610, USA. Tel.: +1 352273 6852; fax: +1 3522736849 .

E-mail address: grantmcf@ufl.edu (G. McFadden).
}

tebrate and insect hosts, respectively (Buller et al., 2005). Characteristic features of poxviruses include a cytoplasmic life cycle, a large virion size and large genome compared to other viruses (Moss, 2007). Poxviruses contain a linear, double-stranded DNA genome with palindromic, covalentlyclosed ends. Sequenced poxvirus genomes vary from $\sim 134$ to $\sim 360 \mathrm{kbp}$ in length and 130 to 328 open reading frames (ORFs) can be predicted from the sequences. At the ends of poxvirus DNA genomes are mirror image terminal inverted repeat (TIR) regions, however, among different strains the lengths of the TIR regions vary from a few hundred nucleotides, such as in Variola virus (VARV), to approximately $12 \mathrm{kbp}$, such as in Shope fibroma virus (SHFV). In general, the chordopoxvirus 
genome is organized so that the essential housekeeping genes, including those required for transcription, replication and morphogenesis, are located within the central region of the genome. Genes nearer to the DNA ends are generally more variable and encode for a wide variety of functions, including genes dedicated to ensure virus replication within the host by modulating the host innate and adaptive immune response (Seet et al., 2003).

The genomic DNA sequences of over 100 different poxvirus strains have been determined. The particular poxvirus sequences used in the present study are listed in Table 1. All of the sequences used are available through GenBank and two curated poxvirus sites-www.poxvirus.org/ and www.biovirus.org/.

There are two species in the genus Yatapoxvirus: Yaba monkey tumor virus (YMTV) and Tanapox virus (TPV). Both species have caused human infection. A previously sequenced poxvirus, Yaba-like disease virus (YLDV) (Lee et al., 2001), is a TPV from an infected non-human primate (Brunetti et al., 2003; Espana et al., 1971; Esposito and Fenner, 2001; McNulty et al., 1968). TPV and YLDV are suspected to be transmitted by arthropod vectors and both produce a similar rash illness, fever with prodromal symptoms that is followed by the development of few nodular skin lesions (Downie and Espana, 1972; Damon, 2007; Knight et al., 1989). In contrast, YMTV produces a very distinct disease, primarily in non-human primates, which is characterized by epidermal histiocytomas of the head and limbs (Downie and Espana, 1972; Knight et al., 1989). The observed biological differences between YMTV and YLDV are likely explained by the $82 \%$ nucleotide identity and an approximately $10 \mathrm{kbp}$ deletion from YMTV compared to YLDV (Brunetti et al., 2003; Downie and Espana, 1972; Espana et al., 1971; Knight et al., 1989; Lee et al., 2001).

A previous study, in which the genome of YMTV was sequenced, examined the conservation of certain gene families that were found to be below the usual 50 codon cutoff (Brunetti et al., 2003). To further this research, two isolates of TPV were sequenced; one is the first isolate from a 1957 human outbreak of TPV in the Tana River valley in Kenya (TPV-Kenya) and the other was isolated from an infected college student traveling in the Congo Basin in the Republic of Congo (TPVRoC) (Dhar et al., 2004). The current study is a comparative genomic analysis of these two isolates of TPV that are from discrete geographic regions of Africa and isolated 50 years apart.

Table 1

Summary information of poxvirus sequences used in this study

\begin{tabular}{|c|c|c|c|c|c|c|}
\hline Genus & Virus & Strain & Virus short form & Genome size (bp) & Accession number & Reference \\
\hline \multirow{4}{*}{ Yatapox } & Tanapox virus & RoC & TPV-RoC & 144553 & EF420157 & This study \\
\hline & Tanapox virus & Kenya & TPV-Kenya & 144565 & EF420156 & This study \\
\hline & Yaba-like disease virus & & YLDV & 144575 & NC_002642 & Lee et al. (2001) \\
\hline & Yaba monkey tumor virus & Roswell Park-Yohn & YMTV & 134721 & NC_005179 & Brunetti et al. (2003) \\
\hline \multirow{3}{*}{ Capripox } & Goatpox virus & Pellor & GTPV & 149599 & NC_004003 & Tulman et al. (2002) \\
\hline & Lumpy skin disease virus & Neethling 2490 & LSDV & 150773 & NC_003027 & Tulman et al. (2001) \\
\hline & Sheeppox virus & A & SHPV & 150057 & AY077833 & Tulman et al. (2002) \\
\hline Suipox & Swinepox virus & Nebraska 17077-99 & SWPV & 146454 & NC_003389 & Afonso et al. $(2002)^{\mathrm{a}}$ \\
\hline \multirow{2}{*}{ Leporipox } & Myxoma virus & Lausanne & MYXV & 161773 & NC_001132 & Cameron et al. (1999) \\
\hline & Shope fibroma virus & Kasza & SHFV & 159857 & NC_001266 & Willer et al. (1999) \\
\hline Molluscipox & Molluscum contagiosum virus & Subtype 1 & MOCV & 190289 & NC_001731 & Senkevich et al. (1996) \\
\hline \multirow{9}{*}{ Orthopox } & Camelpox virus & CMS & CMPV & 202205 & AY009089 & \multirow[t]{3}{*}{ Gubser and Smith (2002) } \\
\hline & Cowpox virus & Brighton Red & CPXV & 224499 & C_003663 & \\
\hline & Ectromelia virus & Moscow & ECTV & 209771 & NC_004105 & \\
\hline & Horsepox virus & MNR-76 & HSPV & 212633 & DQ792504 & Tulman et al. (2006) \\
\hline & Monkeypox virus & Zaire & MPXV & 196858 & NC_003310 & Shchelkunov et al. (2001) \\
\hline & Raccoonpox virus & & RCNV & $\mathrm{ND}^{\mathrm{a}}$ & M23018 & \\
\hline & Taterapox virus & Dahomey 1968 & TATV & 198050 & NC_008291 & \\
\hline & Vaccinia virus & Western Reserve & VACV & 194711 & NC_006998 & \\
\hline & Variola virus & India 19647125 Vellor & VARV & 186127 & DQ437586 & Esposito et al. (2006) \\
\hline \multirow{2}{*}{ Avipox } & Canarypox virus & ATCC VR111 & CNPV & 359853 & NC_005309 & Tulman et al. (2004) \\
\hline & Fowlpox virus & Iowa & FWPV & 288539 & NC_002188 & Afonso et al. (2000) \\
\hline \multirow{2}{*}{ Parapox } & Bovine papular stomatitis virus & BV-AR02 & BPSV & 134431 & NC_005337 & Delhon et al. (2004) \\
\hline & Orf virus & NZ2 & ORFV & 137820 & DQ184476 & Mercer et al. (2006) \\
\hline \multirow{2}{*}{ Unclassified } & Crocodilepox virus & & CRV & 190054 & NC_008030 & Afonso et al. (2006) \\
\hline & Deerpox virus & W-1170-84 & DPV & 170560 & AY689437 & Afonso et al. (2005) \\
\hline
\end{tabular}

\footnotetext{
a Not determined.
} 
Comparative genomics reported here reveals the similarities and differences within and without Yatapoxviruses. In particular, the genetic relationships of TPV with sequenced isolates of the genera Capripoxvirus and Suipoxvirus and an unclassified Deerpox virus are explored.

\section{Materials and methods}

\subsection{Genomic sequencing}

Sequencing was performed essentially as described elsewhere (Esposito et al., 2006). Briefly, TPV genomic DNA was extracted from cells infected with TPV-Kenya (Knight et al., 1989) and TPV-RoC (Dhar et al., 2004). The genomic DNA was used as template for production of a set of 14 overlapping polymerase chain reaction (PCR) amplicons that span virtually the entire viral genome. Amplicons of $10-12 \mathrm{kbp}$ each were produced using the Expand High Fidelity PCR System (Roche Applied Science, Indianapolis, IN, USA). The product of eight identical PCR mixtures for each amplicon were pooled and treated with ExoSap-IT (USB Corporation, Cleveland, $\mathrm{OH}$, USA) to reduce PCR errors in the amplicon templates, which were used for primer-walking cycle-sequencing reactions. Cycle sequencing reactions used Applied Biosystems (PE Biosystems, Foster City, CA, USA) Big-Dye 3.1 dye chemistry and ABI
3730XL DNA sequencers and the sequencing primers (Integrated DNA Technologies, Coralville, IA, USA) were designed to anneal approximately at every 400 bases across the templates, which enabled a nine-fold average sequence redundancy. To verify certain sequences, additional cycle sequencing involved direct sequencing from the full-length extracted genome DNA. Chromatogram data was assembled using Seqmerge (Wisconsin Package Version 10.3, Accelrys Inc., San Diego, CA, USA) and Phred/Phrap base-calling and assembly software and Consed for sequence editing (Balbas and Gosset, 2001; Domi and Moss, 2002). ORFs were identified and alignments performed using MacVector 6.5.3 (Oxford Molecular Ltd.).

\subsection{Estimation of nucleotide substitution}

TPV-Kenya and TPV-RoC were compared 25,000 bp at a time by using a base-by-base pairwise comparison matrix containing 144,565 nucleotide positions. Nucleotide differences were analyzed for transversions and transitions.

\section{Results}

Two TPV isolates from infected humans either living (TPVKenya) or traveling (TPV-RoC) through equatorial Africa were sequenced, which provided an opportunity to investigate the evo-

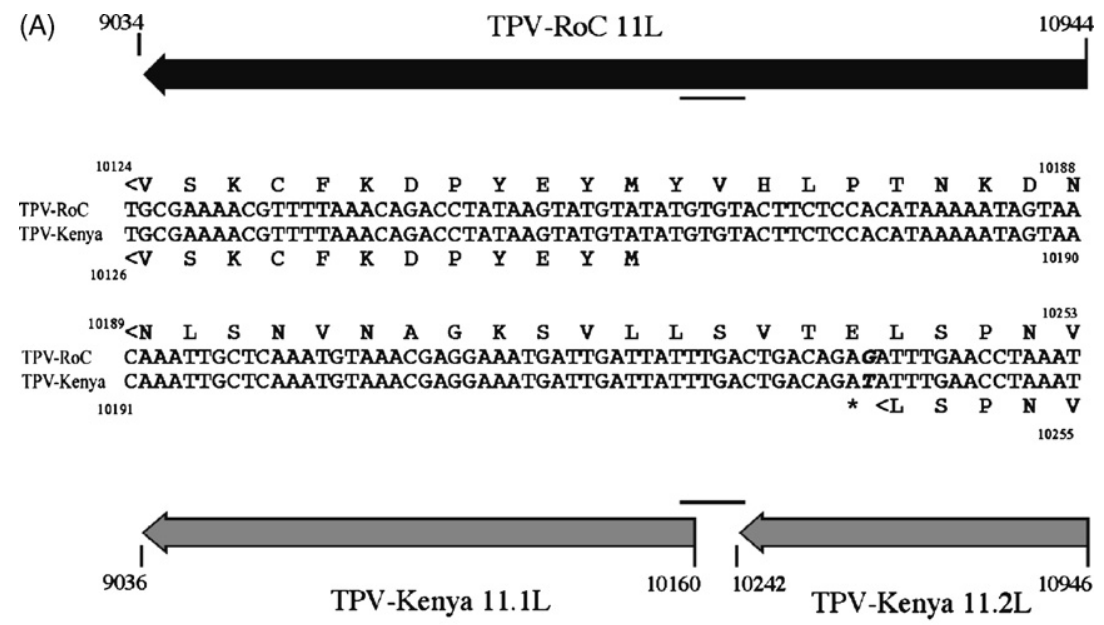

(B)

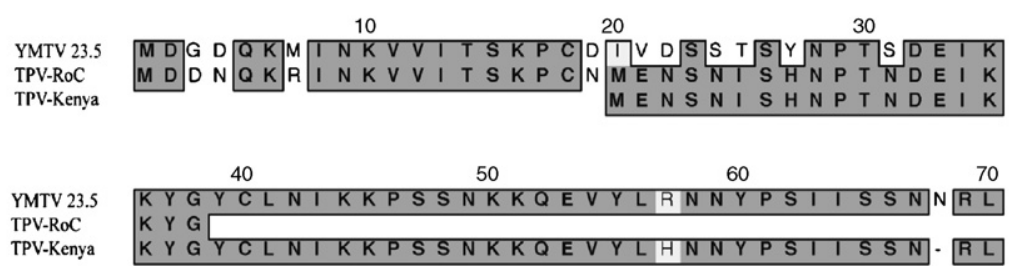

Fig. 1. ORF differences between TPV-RoC and TPV-Kenya. (A) The 11L gene from TPV-RoC (solid black arrow) is a single ORF. The same region from TPV-Kenya encodes two ORFs (solid gray arrows). The sequences between the comparable regions are identical except for a single transversion at position 10239 of TPV-RoC. This change results in a termination codon $(*[T A G])$ in the predicted transcript of TPV-Kenya instead of the incorporation of a glutamic acid (E [GAG]) as predicted for TPV-RoC. The single nucleotide change is bolded and italized. The sequence presented represents the minus strand. The predicted amino acid sequences for TPV-RoC or TPV-Kenya are indicated above or below the corresponding nucleotide sequence. Numbers indicate position with the respective genomes. The single black lines above or below the solid arrows show the region that is represented by the sequence comparison. (B) ClustalW alignment of the 23.5L ORF from YMTV, TPV-Kenya and TPV-RoC. Similar amino acids are shaded light grey and identical amino acids are shaded dark grey. 
lutionary diversity of two TPVs that spanned 50 years and were from two different African countries.

\subsection{Genome architecture of TPV}

In GenBank there are sequences of TPV-Kenya that represent approximately $8 \mathrm{kbp}$ of the total genome (GenBank accession numbers AY253325, AF245394 and AF153912); these sequences are about $98 \%$ identical to cognate sequences in a reported YLDV genome sequence (Lee et al., 2001). In order to sequence the two TPV isolates described here, PCR amplicon and cycle sequencing primers were designed by using the reported YLDV sequence. The determined sequences of TPV-Kenya and TPV-RoC comprised 144,565 and 144,553 bp, respectively, $96 \%$ of which encode for putative ORFs. Both viruses are $73 \% \mathrm{~A}+\mathrm{T}$-rich, which is consistent with the other sequenced yatapoxviruses (YLDV 73\% A + T and YMTV 70\% A + T) (Brunetti et al., 2003; Lee et al., 2001). By comparison with the YLDV sequences, the TPV sequences lack the putative concatemer resolution domain proximal to the hairpin-loop termini. However, the two TPV isolates were sequenced to within
20 bp of cognate reported YLDV genomic sequences (Lee et al., 2001).

TPV-Kenya and TPV-RoC encode 156 and 155 distinct ORFs, respectively (Table 2). All ORFs that were reported for YLDV are present in both isolates of TPV, with two exceptions-ORF 11L and ORF 23.5L (Fig. 1). TPV-Kenya 11L has a premature stop codon at codon 236 , which results in a truncated ORF (Fig. 1A). Approximately $80 \mathrm{bp}$ downstream of the $11 \mathrm{~L}$ stop codon in TPV-Kenya, a putative ORF corresponding to the second half of the $11 \mathrm{~L} \mathrm{ORF}$ is present and may be transcribed as a distinct gene product. The two ORFs in TPV-Kenya are denoted 11.1L and 11.2L (Fig. 1A and Table 2). The two predicted ORFs have been identified previously and were annotated in GenBank. TPV-Kenya 11.1L was previously labeled TPV ORFL7R (accession number AAD46181) and TPV ORFL8R (accession number AAD46182). The ORF 11.2L is identical to TPV ORFL4R (accession number AAD46179), which indicates that this truncated ORF has been independently identified. In contrast, the $11 \mathrm{~L} \mathrm{ORF}$ in TPV-RoC is not truncated. The TPV-RoC 11L-encoded protein is an ankyrin repeat protein that contains a predicted F-box domain (Fig. 2B) (Mercer et

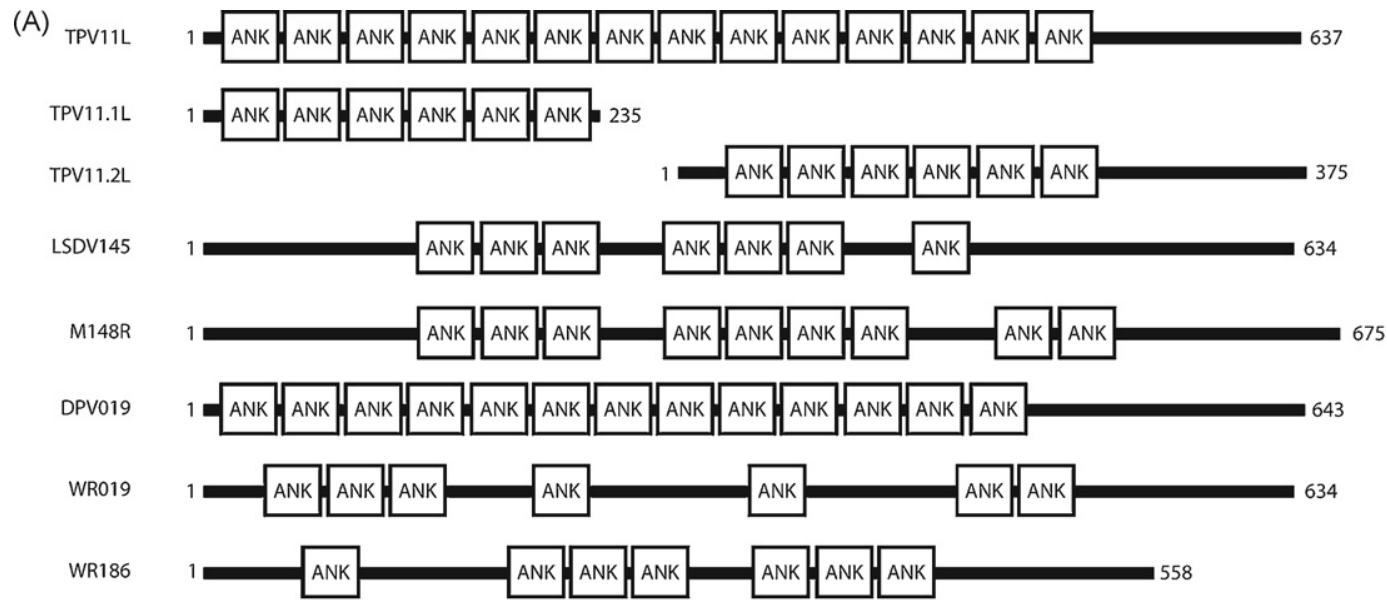

(B)

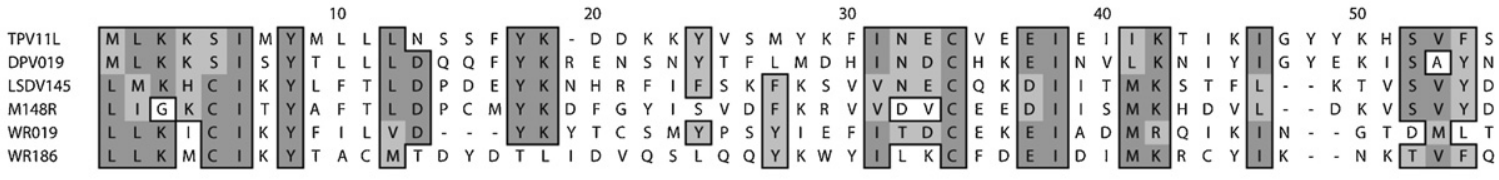
60

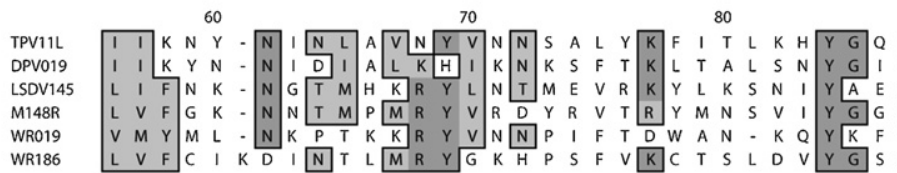

WR186

TPV11L

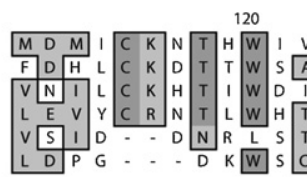

130

140
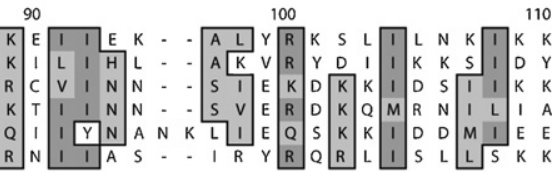

DPV019

LSDV14

M148R
WR019

WR186

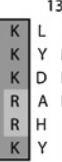

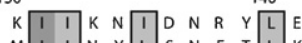

1. $N$ N

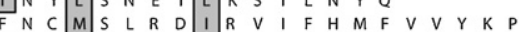

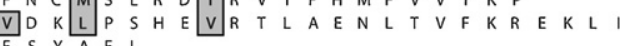

D N E L L T Y L K I L

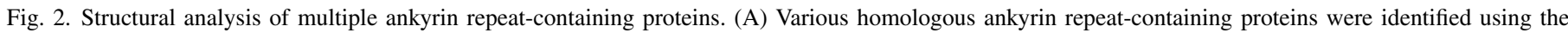

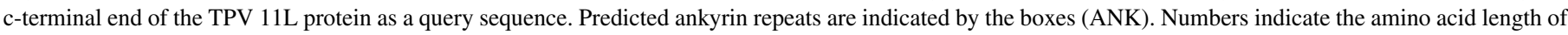

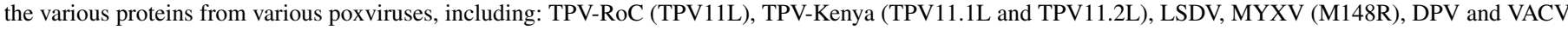

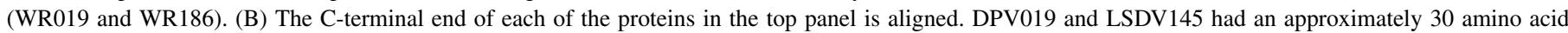

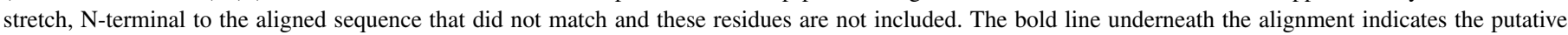
F-box domain that is complete in all sequences but WR019. 
Table 2

Identification of the predicted open reading frames (ORFs) of TPV-Kenya and TPV-RoC

\begin{tabular}{|c|c|c|c|c|c|c|c|c|c|c|c|c|c|c|c|c|c|}
\hline \multirow[t]{3}{*}{ ORF } & \multicolumn{3}{|c|}{ TPV-Kenya } & \multicolumn{3}{|c|}{ TPV-RoC } & \multirow{3}{*}{$\begin{array}{l}\text { Predicted structure } \\
\text { or function }\end{array}$} & \multicolumn{2}{|l|}{$\mathrm{DPV}^{\mathrm{a}}$} & \multicolumn{2}{|l|}{ LSDV $^{\mathrm{b}}$} & \multicolumn{2}{|l|}{$\mathrm{VACV}^{\mathrm{c}}$} & \multicolumn{2}{|l|}{$\mathrm{MYXV}^{\mathrm{d}}$} & \multicolumn{2}{|c|}{$\mathrm{YMTV}^{\mathrm{e}}$} \\
\hline & \multicolumn{2}{|l|}{ Codon } & \multirow[t]{2}{*}{$a a^{f}$} & \multicolumn{2}{|l|}{ Codon } & \multirow[t]{2}{*}{ aa } & & \multirow[t]{2}{*}{ ORF } & \multirow{2}{*}{$\begin{array}{l}\text { Identity/ } \\
\text { similarity }\end{array}$} & \multirow[t]{2}{*}{ ORF } & \multirow{2}{*}{$\begin{array}{l}\text { Identity/ } \\
\text { similarity }\end{array}$} & \multirow[t]{2}{*}{ ORF } & \multirow{2}{*}{$\begin{array}{l}\text { Identity/ } \\
\text { similarity }\end{array}$} & \multirow[t]{2}{*}{ ORF } & \multirow{2}{*}{$\begin{array}{l}\text { Identity/ } \\
\text { similarity }\end{array}$} & \multirow[t]{2}{*}{ ORF } & \multirow{2}{*}{$\begin{array}{l}\text { Identity/ } \\
\text { similarity }\end{array}$} \\
\hline & Start & Stop & & Start & Stop & & & & & & & & & & & & \\
\hline $1 \mathrm{~L}$ & 1738 & 740 & 333 & 1738 & 740 & 333 & & DPV007 & $38 / 60$ & LSDV007 & $33 / 55$ & WR010 & $28 / 49$ & & & $1 \mathrm{~L}$ & $71 / 84$ \\
\hline $2 \mathrm{~L}$ & 2868 & 1855 & 338 & 2868 & 1855 & 338 & $\begin{array}{l}\text { TNF binding } \\
\text { protein }\end{array}$ & DPV008 & $38 / 53$ & & & & & & & $2 \mathrm{~L}$ & $73 / 82$ \\
\hline $3 \mathrm{~L}$ & 3583 & 2918 & 222 & 3583 & 2918 & 222 & & DPV009 & $42 / 66$ & LSDV150 & & WR039 & $38 / 60$ & & & & \\
\hline $4 \mathrm{~L}$ & 4329 & 3616 & 238 & 4329 & 3616 & 238 & & DPV011 & $35 / 62$ & LSDV009 & $35 / 56$ & WR029 & $33 / 62$ & & & $4 \mathrm{~L}$ & $71 / 83$ \\
\hline $5 \mathrm{~L}$ & 4840 & 4373 & 156 & 4840 & 4373 & 156 & LAP/PHD domain & & & LSDV010 & $45 / 59$ & & & M153R & $39 / 56$ & $5 \mathrm{~L}$ & $64 / 82$ \\
\hline $6 \mathrm{~L}$ & 5354 & 4908 & 149 & 5353 & 4907 & 149 & & & & LSDV001 & $37 / 57$ & WR196 & $41 / 63$ & & & $6 \mathrm{~L}$ & $81 / 93$ \\
\hline $7 \mathrm{~L}$ & 6473 & 5421 & 351 & 6471 & 5419 & 351 & $\begin{array}{l}\text { Chemokine } \\
\text { inhibitor }\end{array}$ & DPV013 & $49 / 70$ & LSDV011 & $40 / 66$ & & & & & $7 \mathrm{~L}$ & $74 / 87$ \\
\hline $8 \mathrm{~L}$ & 7131 & 6493 & 213 & 7129 & 6491 & 213 & Ankyrin repeat & DPV014 & $32 / 58$ & LSDV012 & $43 / 65$ & & & M149R & $28 / 53$ & $11 \mathrm{~L}$ & $27 / 53$ \\
\hline 9L & 7848 & 7171 & 226 & 7846 & 7169 & 226 & $\begin{array}{l}\text { Virulence gene } \\
\text { factor }\end{array}$ & DPV017 & $39 / 62$ & & & WR031 & $34 / 51$ & M154L & $26 / 46$ & & \\
\hline $10 \mathrm{~L}$ & 9021 & 7873 & 383 & 9019 & 7871 & 383 & SERPIN/Spi3ortholog & DPV018 & $45 / 61$ & & & WR034 & $29 / 48$ & M008.1 & $31 / 52$ & & \\
\hline $11 \mathrm{~L}$ & & & & 10944 & 9034 & 637 & Ankyrin repeat & DPV019 & $51 / 69$ & & & WR186 & $27 / 45$ & & & $11 \mathrm{~L}$ & $79 / 93$ \\
\hline $11.1 \mathrm{~L}$ & 10160 & 9036 & 375 & & & & Ankyrin repeat & DPV019 & $51 / 69$ & LSDV148 & $23 / 45$ & WR186 & $27 / 46$ & M149R & $29 / 49$ & $11 \mathrm{~L}$ & $79 / 93$ \\
\hline $11.2 \mathrm{~L}$ & 10946 & 10242 & 235 & & & & Ankyrin repeat & DPV019 & $52 / 73$ & LSDV145 & $31 / 52$ & WR019 & $29 / 47$ & M148R & $23 / 45$ & $11 \mathrm{~L}$ & $82 / 94$ \\
\hline $12 \mathrm{~L}$ & 11232 & 10969 & 88 & 11230 & 10967 & 88 & $\begin{array}{l}\text { IF2 } \alpha \text {-like PKR } \\
\text { inhibitor }\end{array}$ & DPV020 & $51 / 73$ & LSDV014 & $47 / 66$ & WR034 & $37 / 57$ & & & $12 \mathrm{~L}$ & $75 / 88$ \\
\hline $13 \mathrm{~L}$ & 12121 & 11267 & 285 & 12119 & 11265 & 285 & $\begin{array}{l}\text { Monoglyceride } \\
\text { lipase }\end{array}$ & & & & WR038 & & $50 / 70$ & & & $13 \mathrm{~L}$ & $76 / 87$ \\
\hline $14 \mathrm{~L}$ & 12554 & 12147 & 136 & 12552 & 12145 & 136 & $\begin{array}{l}\text { IL-18 binding } \\
\text { protein }\end{array}$ & DPV021 & $46 / 68$ & LSDV015 & $40 / 65$ & & & & & $14 \mathrm{~L}$ & $55 / 72$ \\
\hline $15 \mathrm{~L}$ & 12815 & 12585 & 77 & 12813 & 12583 & 77 & $\begin{array}{l}\text { EGF-like growth } \\
\text { factor }\end{array}$ & & & LSDV016 & $41 / 55$ & & & M010L & $36 / 52$ & & \\
\hline $16 \mathrm{~L}$ & 13345 & 12818 & 176 & 13343 & 12816 & 176 & $\begin{array}{l}\text { Mitochondria } \\
\text { anti-apoptotic } \\
\text { factor }\end{array}$ & DPV022 & $35 / 59$ & LSDV017 & $30 / 54$ & & & M011L & $27 / 47$ & $16 \mathrm{~L}$ & $64 / 80$ \\
\hline $17 \mathrm{~L}$ & 13821 & 13393 & 143 & 13819 & 13391 & 143 & dUTPase & DPV023 & $62 / 74$ & LSDV018 & $64 / 76$ & WR041 & $58 / 73$ & M012L & $63 / 80$ & $17 \mathrm{~L}$ & $86 / 95$ \\
\hline $18 \mathrm{~L}$ & 14246 & 13866 & 127 & 14244 & 13864 & 127 & Pyrin domain & DPV024 & $40 / 59$ & & & & & M013L & $30 / 55$ & & \\
\hline 19L & 15846 & 14281 & 522 & 15844 & 14279 & 522 & Kelch protein & DPV025 & $37 / 62$ & LSDV019 & $34 / 54$ & WR042 & $27 / 48$ & M014L & $24 / 57$ & $19 \mathrm{~L}$ & $76 / 89$ \\
\hline $20 \mathrm{~L}$ & 16848 & 15874 & 325 & 16846 & 15872 & 325 & $\begin{array}{l}\text { Ribonucleotide } \\
\text { reductase }\end{array}$ & DPV026 & $77 / 89$ & LSDV020 & $78 / 89$ & WR043 & $77 / 87$ & M015L & $75 / 86$ & $20 \mathrm{~L}$ & $91 / 95$ \\
\hline $21 \mathrm{~L}$ & 17133 & 16879 & 85 & 17131 & 16877 & 85 & & DPV027 & $30 / 61$ & LSDV021 & $38 / 60$ & & & M016L & $42 / 66$ & $21 \mathrm{~L}$ & $65 / 84$ \\
\hline $22 \mathrm{~L}$ & 17480 & 17172 & 103 & 17478 & 17170 & 103 & & & & & & & & & & $22 \mathrm{~L}$ & $34 / 55$ \\
\hline $23 \mathrm{~L}$ & 17703 & 17485 & 73 & 17701 & 17483 & 73 & & & & & & & & & & & \\
\hline $23.5 \mathrm{~L}$ & 17949 & 17800 & 50 & 18005 & 17892 & 38 & & DPV029 & $59 / 68$ & LSDV023 & $68 / 85$ & WR047 & $43 / 69$ & M018L & $58 / 76$ & $23.5 \mathrm{~L}$ & $86 / 95$ \\
\hline $24 \mathrm{~L}$ & 18721 & 18080 & 214 & 18719 & 18078 & 214 & & DPV031 & $56 / 82$ & LSDV024 & $50 / 73$ & WR048 & $52 / 75$ & M019L & $46 / 73$ & $24 \mathrm{~L}$ & $84 / 93$ \\
\hline $25 \mathrm{~L}$ & 20036 & 18702 & 445 & 20034 & 18700 & 445 & $\begin{array}{l}\text { Serine/threonine } \\
\text { protein kinase }\end{array}$ & DPV032 & $77 / 90$ & LSDV025 & $78 / 90$ & WR049 & $72 / 83$ & M020L & $73 / 87$ & $25 \mathrm{~L}$ & $90 / 96$ \\
\hline $26 \mathrm{~L}$ & 21988 & 20063 & 642 & 21986 & 20061 & 642 & EEV maturation & DPV034 & $48 / 69$ & LSDV027 & $45 / 61$ & WR051 & $36 / 56$ & M021L & $41 / 61$ & $26 \mathrm{~L}$ & $70 / 86$ \\
\hline $27 \mathrm{~L}$ & 23133 & 22024 & 370 & 23131 & 22022 & 370 & $\begin{array}{l}\text { Palmitylated EEV } \\
\text { envelope protein }\end{array}$ & DPV035 & $72 / 86$ & LSDV028 & $77 / 87$ & WR052 & $58 / 74$ & M022L & $72 / 83$ & $27 \mathrm{~L}$ & $90 / 94$ \\
\hline $28 \mathrm{~L}$ & 23332 & 23189 & 48 & 23330 & 23187 & 48 & & & & & & & & & & & \\
\hline $28.5 \mathrm{~L}$ & 23529 & 23359 & 57 & 23527 & 23357 & 57 & & & & & & & & & & $28.5 \mathrm{~L}$ & $67 / 84$ \\
\hline
\end{tabular}




\begin{tabular}{|c|c|c|c|c|c|c|c|c|c|c|c|c|c|c|c|c|c|}
\hline \multirow[t]{3}{*}{ ORF } & \multicolumn{3}{|c|}{ TPV-Kenya } & \multicolumn{3}{|c|}{ TPV-RoC } & \multirow{3}{*}{$\begin{array}{l}\text { Predicted structure } \\
\text { or function }\end{array}$} & \multicolumn{2}{|l|}{$\mathrm{DPV}^{\mathrm{a}}$} & \multicolumn{2}{|l|}{$\mathrm{LSDV}^{\mathrm{b}}$} & \multicolumn{2}{|l|}{$\mathrm{VACV}^{\mathrm{c}}$} & \multicolumn{2}{|l|}{ MYXV $^{\mathrm{d}}$} & \multicolumn{2}{|c|}{ YMTV $^{\mathrm{e}}$} \\
\hline & \multicolumn{2}{|l|}{ Codon } & \multirow[t]{2}{*}{$a a^{f}$} & \multicolumn{2}{|l|}{ Codon } & \multirow[t]{2}{*}{ aa } & & \multirow[t]{2}{*}{ ORF } & \multirow{2}{*}{$\begin{array}{l}\text { Identity/ } \\
\text { similarity }\end{array}$} & \multirow[t]{2}{*}{ ORF } & \multirow{2}{*}{$\begin{array}{l}\text { Identity/ } \\
\text { similarity }\end{array}$} & \multirow[t]{2}{*}{ ORF } & \multirow{2}{*}{$\begin{array}{l}\text { Identity/ } \\
\text { similarity }\end{array}$} & \multirow[t]{2}{*}{ ORF } & \multirow{2}{*}{$\begin{array}{l}\text { Identity/ } \\
\text { similarity }\end{array}$} & ORF & Identity/ \\
\hline & Start & Stop & & Start & Stop & & & & & & & & & & & & the \\
\hline $29 \mathrm{~L}$ & 24027 & 23584 & 148 & 24025 & 23582 & 148 & & DPV037 & $66 / 80$ & LSDV029 & $67 / 81$ & WR054 & $56 / 73$ & M024L & $52 / 72$ & $29 \mathrm{~L}$ & $82 / 92$ \\
\hline $30 \mathrm{~L}$ & 24738 & 24094 & 215 & 24736 & 24092 & 215 & & DPV038 & $41 / 61$ & LSDV030 & $38 / 59$ & WR055 & $41 / 59$ & M025L & $33 / 55$ & $30 \mathrm{~L}$ & $77 / 92$ \\
\hline $31 \mathrm{R}$ & 24797 & 25111 & 105 & 24795 & 25109 & 105 & $\begin{array}{l}\text { DNA-binding } \\
\text { virion core protein }\end{array}$ & DPV039 & $70 / 86$ & LSDV031 & $73 / 83$ & WR056 & $55 / 75$ & M026R & $70 / 83$ & $31 \mathrm{R}$ & $90 / 95$ \\
\hline $32 \mathrm{~L}$ & 26523 & 25114 & 470 & 26521 & 25112 & 470 & $\begin{array}{l}\text { Poly(A) } \\
\text { polymerase }\end{array}$ & DPV040 & $72 / 86$ & LSDV032 & $72 / 85$ & WR057 & $67 / 83$ & M027L & $71 / 84$ & $32 \mathrm{~L}$ & $91 / 98$ \\
\hline $33 \mathrm{~L}$ & 28597 & 26543 & 685 & 28595 & 26541 & 685 & & DPV041 & $50 / 72$ & LSDV033 & $44 / 66$ & WR058 & $40 / 62$ & M028L & $45 / 66$ & $33 \mathrm{~L}$ & $72 / 88$ \\
\hline $34 \mathrm{~L}$ & 29202 & 28660 & 181 & 29200 & 28658 & 181 & dsRNA-binding & DPV042 & $45 / 64$ & LSDV034 & $49 / 69$ & WR059 & $38 / 55$ & M029L & $58 / 75$ & $34 \mathrm{~L}$ & $68 / 83$ \\
\hline $35 \mathrm{~L}$ & 29811 & 29245 & 189 & 29809 & 29243 & 189 & $\begin{array}{l}\text { RNA polymerase } \\
\text { subunit rpo30 }\end{array}$ & DPV043 & $68 / 83$ & LSDV036 & $69 / 81$ & WR060 & $71 / 85$ & M030L & $67 / 82$ & $35 \mathrm{~L}$ & $86 / 93$ \\
\hline $36 \mathrm{R}$ & 29928 & 30983 & 352 & 29926 & 30981 & 352 & & DPV044 & $35 / 51$ & LSDV035 & $37 / 56$ & WR061 & $25 / 44$ & M031R & $31 / 53$ & $36 \mathrm{R}$ & $74 / 88$ \\
\hline $37 \mathrm{R}$ & 30987 & 32687 & 567 & 30985 & 32685 & 567 & & DPV045 & $70 / 85$ & LSDV037 & $70 / 85$ & WR062 & $61 / 81$ & M032R & $63 / 81$ & $37 \mathrm{R}$ & $87 / 95$ \\
\hline $38 \mathrm{R}$ & 32710 & 33513 & 268 & 32708 & 33511 & 268 & & DPV046 & $80 / 91$ & LSDV038 & 79/91 & WR064 & $70 / 81$ & M033R & $76 / 87$ & $38 \mathrm{R}$ & 93/97 \\
\hline $39 \mathrm{~L}$ & 36533 & 33516 & 1006 & 36531 & 33514 & 1006 & DNA polymerase & DPV047 & $70 / 84$ & LSDV039 & $70 / 85$ & WR065 & $69 / 82$ & M034R & $70 / 84$ & $39 \mathrm{~L}$ & $86 / 93$ \\
\hline $40 \mathrm{R}$ & 36566 & 36847 & 94 & 36564 & 36845 & 94 & & DPV048 & $70 / 88$ & LSDV040 & $72 / 86$ & WR066 & $66 / 80$ & M035R & $66 / 89$ & $40 \mathrm{R}$ & $88 / 97$ \\
\hline $41 \mathrm{~L}$ & 37236 & 36850 & 129 & 37234 & 36848 & 129 & & & & LSDV041 & $54 / 74$ & WR067 & $44 / 71$ & & & $41 \mathrm{~L}$ & $74 / 87$ \\
\hline $42 \mathrm{~L}$ & 39262 & 37226 & 679 & 39260 & 37224 & 679 & & DPV050 & $49 / 69$ & LSDV042 & $42 / 65$ & WR068 & $39 / 61$ & M036L & $41 / 65$ & & \\
\hline $42.5 \mathrm{~L}$ & 39413 & 39324 & 30 & 39411 & 39322 & 30 & & & & & & & & M037L & $67 / 82$ & & \\
\hline $43 \mathrm{~L}$ & 40365 & 39433 & 311 & 40363 & 39431 & 311 & $\begin{array}{l}\text { DNA binding core } \\
\text { protein }\end{array}$ & DPV051 & $73 / 87$ & LSDV043 & $69 / 86$ & WR070 & $68 / 83$ & M038L & $69 / 87$ & $43 \mathrm{~L}$ & $86 / 96$ \\
\hline $44 \mathrm{~L}$ & 40590 & 40369 & 74 & 40588 & 40367 & 74 & & DPV052 & $50 / 63$ & LSDV044 & $49 / 66$ & WR071 & $45 / 62$ & M039L & $45 / 68$ & $44 \mathrm{~L}$ & $79 / 89$ \\
\hline $45 \mathrm{~L}$ & 41391 & 40594 & 266 & 41389 & 40592 & 266 & $\begin{array}{l}\text { ssDNA-binding } \\
\text { phosphoprotein }\end{array}$ & DPV053 & $64 / 83$ & LSDV045 & $61 / 77$ & WR072 & $55 / 72$ & M040L & $58 / 81$ & $45 \mathrm{~L}$ & $87 / 94$ \\
\hline $46 \mathrm{~L}$ & 41694 & 41458 & 79 & 41692 & 41456 & 79 & Structural & DPV055 & 78/93 & LSDV046 & $68 / 81$ & WR074 & $46 / 76$ & M041L & $46 / 71$ & $46 \mathrm{~L}$ & $86 / 96$ \\
\hline $47 \mathrm{~L}$ & 42872 & 41715 & 386 & 42870 & 41713 & 386 & & DPV056 & $55 / 78$ & LSDV047 & $54 / 72$ & WR075 & $54 / 74$ & M042L & $50 / 71$ & $47 \mathrm{~L}$ & $83 / 93$ \\
\hline $48 \mathrm{~L}$ & 44158 & 42872 & 429 & 44156 & 42870 & 429 & Topoisomerase II & DPV057 & $77 / 90$ & LSDV048 & $76 / 88$ & WR076 & $69 / 85$ & M043L & $73 / 85$ & $48 \mathrm{~L}$ & $92 / 97$ \\
\hline $49 \mathrm{R}$ & 44164 & 46191 & 676 & 44162 & 46189 & 676 & Helicase & DPV058 & $62 / 78$ & LSDV049 & $65 / 80$ & WR077 & $58 / 76$ & M044R & $57 / 75$ & $49 \mathrm{R}$ & $84 / 93$ \\
\hline $50 \mathrm{~L}$ & 47963 & 46194 & 590 & 47961 & 46192 & 590 & Metalloprotease & DPV059 & $64 / 79$ & LSDV050 & $60 / 78$ & WR078 & $55 / 75$ & M45L & $58 / 75$ & $50 \mathrm{~L}$ & $82 / 92$ \\
\hline $51 \mathrm{~L}$ & 48295 & 47963 & 111 & 48293 & 47961 & 111 & & DPV061 & $55 / 75$ & LSDV052 & $43 / 66$ & WR079 & $44 / 65$ & M46L & $54 / 73$ & $51 \mathrm{~L}$ & $73 / 86$ \\
\hline $52 \mathrm{R}$ & 48289 & 48954 & 222 & 48287 & 48952 & 222 & $\begin{array}{l}\text { Transcriptional } \\
\text { elongation factor }\end{array}$ & DPV060 & $50 / 72$ & LSDV051 & $49 / 70$ & WR080 & $49 / 71$ & M47R & $46 / 66$ & $52 \mathrm{R}$ & $85 / 94$ \\
\hline $53 \mathrm{~L}$ & 49301 & 48927 & 125 & 49299 & 48925 & 125 & Glutaredoxin & DPV062 & $73 / 88$ & LSDV053 & $76 / 88$ & WR081 & $44 / 65$ & M48L & $68 / 85$ & $53 \mathrm{~L}$ & $99 / 100$ \\
\hline $54 \mathrm{R}$ & 49304 & 50620 & 439 & 49302 & 50618 & 439 & & DPV063 & $53 / 69$ & LSDV054 & $51 / 70$ & WR082 & $44 / 61$ & M49R & $47 / 67$ & $54 \mathrm{R}$ & $76 / 89$ \\
\hline $55 \mathrm{R}$ & 50626 & 50814 & 63 & 50624 & 50812 & 63 & $\begin{array}{l}\text { RNA polymerase } \\
\text { subunit rpo } 7\end{array}$ & DPV064 & $85 / 93$ & LSDV055 & $85 / 95$ & WR083 & $79 / 88$ & M50R & $85 / 93$ & $55 \mathrm{R}$ & $96 / 98$ \\
\hline $56 \mathrm{R}$ & 50817 & 51335 & 173 & 50815 & 51333 & 173 & & DPV065 & $55 / 77$ & LSDV056 & $56 / 73$ & WR084 & $46 / 70$ & M51R & $55 / 78$ & $56 \mathrm{R}$ & $80 / 89$ \\
\hline $57 \mathrm{~L}$ & 52483 & 51362 & 374 & 52481 & 51360 & 374 & Virion core protein & DPV066 & $64 / 79$ & LSDV057 & $60 / 75$ & WR085 & $51 / 68$ & M52L & $55 / 69$ & $57 \mathrm{~L}$ & $82 / 91$ \\
\hline $58 \mathrm{R}$ & 52513 & 53292 & 260 & 52511 & 53290 & 260 & $\begin{array}{l}\text { Late transcription } \\
\text { factor VLTF-1 }\end{array}$ & DPV067 & $88 / 98$ & LSDV058 & $87 / 97$ & WR086 & $83 / 94$ & M53R & $83 / 94$ & $58 \mathrm{R}$ & $97 / 99$ \\
\hline $59 \mathrm{R}$ & 53308 & 54309 & 334 & 53306 & 54307 & 334 & & DPV068 & $64 / 80$ & LSDV059 & $60 / 77$ & WR087 & $50 / 70$ & M54R & $56 / 74$ & $59 \mathrm{R}$ & $80 / 90$ \\
\hline $60 \mathrm{R}$ & 54313 & 55053 & 247 & 54311 & 55051 & 247 & $\begin{array}{l}\text { IMV membrane } \\
\text { protein }\end{array}$ & DPV069 & $81 / 91$ & LSDV060 & $81 / 93$ & WR088 & $69 / 83$ & M55R & $74 / 92$ & $60 \mathrm{R}$ & $91 / 96$ \\
\hline $61 \mathrm{R}$ & 55075 & 55347 & 91 & 55073 & 55345 & 91 & & DPV070 & $45 / 67$ & LSDV061 & $45 / 70$ & WR089 & $30 / 55$ & M56R & $24 / 50$ & $61 \mathrm{R}$ & $71 / 84$ \\
\hline $62 \mathrm{~L}$ & 56276 & 55329 & 316 & 56274 & 55327 & 316 & & DPV071 & $65 / 83$ & LSDV062 & $66 / 84$ & WR090 & $54 / 74$ & M57L & $60 / 80$ & $62 \mathrm{~L}$ & $86 / 95$ \\
\hline $63 \mathrm{R}$ & 56301 & 57047 & 249 & 56299 & 57045 & 249 & Core protein VP8 & DPV072 & $78 / 87$ & LSDV063 & $78 / 89$ & WR091 & $60 / 82$ & M58R & $76 / 87$ & $63 \mathrm{R}$ & $91 / 95$ \\
\hline
\end{tabular}




\begin{tabular}{|c|c|c|c|c|c|c|c|c|c|c|c|c|c|c|c|c|c|}
\hline $64 \mathrm{R}$ & 57066 & 57449 & 128 & 57064 & 57447 & 128 & $\begin{array}{l}\text { IMV membrane } \\
\text { protein }\end{array}$ & DPV073 & $51 / 75$ & LSDV064 & $59 / 77$ & WR092 & $46 / 67$ & M59R & $52 / 70$ & $64 \mathrm{R}$ & $78 / 90$ \\
\hline $65 \mathrm{R}$ & 57406 & 57882 & 159 & 57404 & 57880 & 159 & Virion protein & DPV074 & $63 / 81$ & LSDV065 & $67 / 78$ & WR093 & $49 / 69$ & M60R & $59 / 71$ & $65 \mathrm{R}$ & $83 / 92$ \\
\hline $66 \mathrm{R}$ & 57882 & 58430 & 183 & 57880 & 58428 & 183 & Thymidine kinase & DPV075 & $67 / 78$ & LSDV066 & $63 / 77$ & WR094 & $70 / 78$ & M61R & $66 / 78$ & $66 \mathrm{R}$ & $82 / 90$ \\
\hline 67R & 58481 & 59014 & 178 & 58479 & 59012 & 178 & Host-range protein & DPV076 & $43 / 67$ & LSDV067 & $42 / 59$ & WR021 & $36 / 66$ & M62R & $42 / 64$ & $67 R$ & $80 / 92$ \\
\hline $68 \mathrm{R}$ & 59084 & 60082 & 333 & 59081 & 60079 & 333 & $\begin{array}{l}\text { Poly-A } \\
\text { polymerase small } \\
\text { subunit }\end{array}$ & DPV077 & $73 / 89$ & LSDV068 & $76 / 90$ & WR095 & $71 / 87$ & M65R & $73 / 87$ & $68 \mathrm{R}$ & $91 / 95$ \\
\hline $69 \mathrm{R}$ & 60000 & 60554 & 185 & 59997 & 60551 & 185 & $\begin{array}{l}\text { RNA polymerase } \\
\text { subunit rpo22 }\end{array}$ & DPV078 & $76 / 87$ & LSDV069 & $77 / 90$ & WR096 & $74 / 87$ & M66R & $71 / 85$ & $69 \mathrm{R}$ & $92 / 95$ \\
\hline $70 \mathrm{~L}$ & 60947 & 60537 & 137 & 60944 & 60534 & 137 & & DPV079 & $67 / 81$ & LSDV070 & $62 / 80$ & WR097 & $61 / 80$ & M67L & $66 / 81$ & $70 \mathrm{~L}$ & $82 / 94$ \\
\hline $71 \mathrm{R}$ & 61038 & 64892 & 1285 & 61035 & 64889 & 1285 & $\begin{array}{l}\text { RNA polymerase } \\
\text { subunit rpo147 }\end{array}$ & DPV080 & $87 / 94$ & LSDV071 & $84 / 93$ & WR098 & $80 / 92$ & M68R & $85 / 94$ & $71 \mathrm{R}$ & 93/97 \\
\hline $72 \mathrm{~L}$ & 65407 & 64895 & 171 & 65404 & 64892 & 171 & $\begin{array}{l}\text { Dual specificity } \\
\text { Ser/Thr and Tyr } \\
\text { phosphatase }\end{array}$ & DPV081 & $81 / 91$ & LSDV072 & $75 / 89$ & WR099 & $63 / 83$ & M69L & $76 / 88$ & $72 \mathrm{~L}$ & $88 / 97$ \\
\hline $73 R$ & 65423 & 65992 & 190 & 65420 & 65989 & 190 & & DPV082 & $69 / 86$ & LSDV073 & $64 / 83$ & WR100 & $62 / 80$ & M70R & $63 / 81$ & $73 \mathrm{R}$ & $84 / 94$ \\
\hline $74 \mathrm{~L}$ & 66969 & 66001 & 323 & 66966 & 65998 & 323 & $\begin{array}{l}\text { IMV envelope } \\
\text { protein p35 }\end{array}$ & DPV083 & $54 / 74$ & LSDV074 & $52 / 73$ & WR101 & $35 / 61$ & M71L & $50 / 74$ & $74 \mathrm{~L}$ & $80 / 91$ \\
\hline $75 \mathrm{~L}$ & 69366 & 66973 & 798 & 69363 & 66970 & 798 & $\begin{array}{l}\text { RNA polymerase- } \\
\text { associated } \\
\text { RAP94 }\end{array}$ & DPV084 & $78 / 89$ & LSDV075 & $77 / 88$ & WR102 & $69 / 84$ & M72L & $75 / 85$ & $75 \mathrm{~L}$ & $91 / 97$ \\
\hline $76 \mathrm{R}$ & 69525 & 70064 & 180 & 69522 & 70061 & 180 & $\begin{array}{l}\text { Late transcription } \\
\text { factor VLTF-4 }\end{array}$ & DPV085 & $48 / 68$ & LSDV076 & $43 / 64$ & WR103 & $41 / 57$ & M73R & $44 / 65$ & $76 \mathrm{R}$ & $77 / 86$ \\
\hline $77 \mathrm{R}$ & 70080 & 71024 & 315 & 70077 & 71021 & 315 & $\begin{array}{l}\text { DNA } \\
\text { topoisomerase }\end{array}$ & DPV086 & $68 / 85$ & LSDV077 & $69 / 86$ & WR104 & $63 / 82$ & M74R & $62 / 82$ & $77 \mathrm{R}$ & $84 / 93$ \\
\hline $78 \mathrm{R}$ & 71043 & 71486 & 148 & 71040 & 71483 & 148 & & DPV087 & $51 / 67$ & LSDV078 & $52 / 69$ & WR105 & $39 / 68$ & M75R & $51 / 69$ & $78 \mathrm{R}$ & $80 / 91$ \\
\hline $79 \mathrm{R}$ & 71526 & 74045 & 840 & 71524 & 74043 & 840 & $\begin{array}{l}\text { mRNA capping } \\
\text { enzyme large } \\
\text { subunit }\end{array}$ & DPV088 & $71 / 87$ & LSDV079 & $72 / 86$ & WR106 & $68 / 84$ & M76R & $69 / 84$ & $79 \mathrm{R}$ & $88 / 95$ \\
\hline $80 \mathrm{~L}$ & 74471 & 74013 & 153 & 74469 & 74011 & 153 & Virion protein & DPV090 & $41 / 64$ & LSDV080 & $36 / 59$ & WR107 & $48 / 68$ & M77L & $40 / 63$ & $80 \mathrm{~L}$ & $74 / 91$ \\
\hline $81 \mathrm{R}$ & 74470 & 75204 & 245 & 74468 & 75202 & 245 & Virion protein & DPV089 & $41 / 61$ & LSDV081 & $36 / 61$ & WR108 & $38 / 56$ & M78R & $30 / 54$ & $81 \mathrm{R}$ & $67 / 84$ \\
\hline $82 \mathrm{R}$ & 75204 & 75857 & 218 & 75202 & 75855 & 218 & $\begin{array}{l}\text { Uracil DNA } \\
\text { glycosylase }\end{array}$ & DPV091 & $76 / 88$ & LSDV082 & $72 / 87$ & WR109 & $69 / 88$ & M79R & $72 / 87$ & $82 \mathrm{R}$ & $82 / 93$ \\
\hline $83 \mathrm{R}$ & 75924 & 78281 & 786 & 75922 & 78279 & 786 & NTPase & DPV092 & $80 / 92$ & LSDV083 & $78 / 91$ & WR110 & $70 / 85$ & M80R & $77 / 90$ & $83 \mathrm{R}$ & $94 / 98$ \\
\hline $84 \mathrm{R}$ & 78281 & 80185 & 635 & 78279 & 80183 & 635 & $\begin{array}{l}\text { Early } \\
\text { transctription } \\
\text { factor VETFs }\end{array}$ & DPV093 & $88 / 94$ & LSDV084 & $88 / 94$ & WR111 & $80 / 90$ & M81R & $86 / 93$ & $84 \mathrm{R}$ & $95 / 99$ \\
\hline $85 \mathrm{R}$ & 80221 & 80700 & 160 & 80219 & 80698 & 160 & $\begin{array}{l}\text { RNA polymerase } \\
\text { subunit rpo18 }\end{array}$ & DPV094 & $77 / 92$ & LSDV085 & $80 / 93$ & WR112 & $71 / 85$ & M82R & $77 / 90$ & $85 \mathrm{R}$ & $94 / 98$ \\
\hline $86 \mathrm{R}$ & 80748 & 81383 & 212 & 80746 & 81381 & 212 & mutT motif & DPV095 & $70 / 84$ & LSDV086 & $68 / 82$ & WR114 & $61 / 77$ & M84R & $58 / 77$ & $86 \mathrm{R}$ & $87 / 95$ \\
\hline $87 \mathrm{R}$ & 81383 & 82147 & 255 & 81381 & 82145 & 255 & mutT motif & DPV096 & $65 / 81$ & LSDV087 & $65 / 80$ & WR115 & $50 / 70$ & M85R & $59 / 78$ & $87 R$ & $89 / 96$ \\
\hline $88 \mathrm{~L}$ & 84038 & 82146 & 631 & 84036 & 82144 & 631 & $\begin{array}{l}\text { Transcription } \\
\text { termination factor } \\
\text { NPH-1 }\end{array}$ & DPV097 & $75 / 89$ & LSDV088 & $75 / 88$ & WR116 & $70 / 86$ & M86L & $71 / 86$ & $88 \mathrm{~L}$ & $92 / 97$ \\
\hline
\end{tabular}




\begin{tabular}{|c|c|c|c|c|c|c|c|c|c|c|c|c|c|c|c|c|c|}
\hline \multirow[t]{3}{*}{ ORF } & \multicolumn{3}{|c|}{ TPV-Kenya } & \multicolumn{3}{|c|}{ TPV-RoC } & \multirow{3}{*}{$\begin{array}{l}\text { Predicted structure } \\
\text { or function }\end{array}$} & \multicolumn{2}{|l|}{$\mathrm{DPV}^{\mathrm{a}}$} & \multicolumn{2}{|l|}{$\mathrm{LSDV}^{\mathrm{b}}$} & \multicolumn{2}{|l|}{$\mathrm{VACV}^{\mathrm{c}}$} & \multicolumn{2}{|l|}{$\mathrm{MYXV}^{\mathrm{d}}$} & \multicolumn{2}{|c|}{ YMTV $^{\mathrm{e}}$} \\
\hline & \multicolumn{2}{|l|}{ Codon } & \multirow[t]{2}{*}{$a a^{f}$} & \multicolumn{2}{|l|}{ Codon } & \multirow[t]{2}{*}{ aа } & & \multirow[t]{2}{*}{$\overline{\mathrm{ORF}}$} & \multirow{2}{*}{$\begin{array}{l}\text { Identity/ } \\
\text { similarity }\end{array}$} & \multirow[t]{2}{*}{$\overline{\mathrm{ORF}}$} & \multirow{2}{*}{$\begin{array}{l}\text { Identity/ } \\
\text { similarity }\end{array}$} & \multirow[t]{2}{*}{$\overline{\mathrm{ORF}}$} & \multirow{2}{*}{$\begin{array}{l}\text { Identity/ } \\
\text { similarity }\end{array}$} & \multirow[t]{2}{*}{ ORF } & \multirow{2}{*}{$\begin{array}{l}\text { Identity/ } \\
\text { similarity }\end{array}$} & \multirow[t]{2}{*}{ ORF } & \multirow{2}{*}{$\begin{array}{l}\text { Identity/ } \\
\text { similarity }\end{array}$} \\
\hline & Start & Stop & & Start & Stop & & & & & & & & & & & & \\
\hline $89 \mathrm{~L}$ & 84933 & 84073 & 287 & 84931 & 84071 & 287 & $\begin{array}{l}\text { mRNA capping } \\
\text { enzyme VITF }\end{array}$ & DPV098 & $80 / 91$ & LSDV089 & $77 / 88$ & WR117 & $74 / 89$ & M87L & $77 / 88$ & $89 \mathrm{~L}$ & $95 / 98$ \\
\hline $90 \mathrm{~L}$ & 86620 & 84965 & 552 & 86618 & 84963 & 552 & $\begin{array}{l}\text { Rifampin } \\
\text { resistance protein }\end{array}$ & DPV099 & $80 / 92$ & LSDV090 & $80 / 91$ & WR118 & $73 / 86$ & M88L & $77 / 90$ & $90 \mathrm{~L}$ & $93 / 97$ \\
\hline $91 \mathrm{~L}$ & 87096 & 86647 & 150 & 87094 & 86645 & 150 & $\begin{array}{l}\text { Late transcription } \\
\text { factor VLTF-2 }\end{array}$ & DPV100 & $64 / 85$ & LSDV091 & $66 / 84$ & WR119 & $63 / 85$ & M89L & $72 / 88$ & $91 \mathrm{~L}$ & $87 / 95$ \\
\hline $92 \mathrm{~L}$ & 87794 & 87123 & 224 & 87792 & 87121 & 224 & $\begin{array}{l}\text { Late transcription } \\
\text { factor VLTF-3 }\end{array}$ & DPV101 & $88 / 95$ & LSDV092 & $84 / 93$ & WR120 & $84 / 95$ & M90L & $86 / 94$ & $92 \mathrm{~L}$ & $95 / 98$ \\
\hline $93 \mathrm{~L}$ & 88018 & 87794 & 75 & 88016 & 87792 & 75 & $\begin{array}{l}\text { Redox virion } \\
\text { protein }\end{array}$ & DPV102 & $60 / 82$ & LSDV093 & $64 / 85$ & WR121 & $55 / 76$ & M91L & $68 / 82$ & $93 \mathrm{~L}$ & $84 / 93$ \\
\hline 94L & 90001 & 88031 & 657 & 89999 & 88029 & 657 & $4 \mathrm{~b}$ core protein & DPV103 & $79 / 90$ & LSDV094 & $73 / 87$ & WR122 & $64 / 80$ & M92L & $75 / 87$ & $94 \mathrm{~L}$ & 93/97 \\
\hline $95 \mathrm{~L}$ & 90516 & 90061 & 152 & 90514 & 90059 & 152 & Virion core protein & DPV104 & $44 / 62$ & LSDV095 & $36 / 55$ & & & M93L & $31 / 55$ & $95 \mathrm{~L}$ & $68 / 82$ \\
\hline 96R & 90556 & 91059 & 168 & 90554 & 91057 & 168 & $\begin{array}{l}\text { RNA polymerase } \\
\text { subunit rpo19 }\end{array}$ & DPV105 & $64 / 81$ & LSDV096 & $68 / 85$ & WR124 & $62 / 78$ & M94R & $62 / 81$ & $96 \mathrm{R}$ & $87 / 94$ \\
\hline 97L & 92174 & 91062 & 371 & 92172 & 91060 & 371 & & DPV106 & $72 / 89$ & LSDV097 & $75 / 87$ & WR125 & $57 / 78$ & M95L & $69 / 86$ & $97 \mathrm{~L}$ & $92 / 98$ \\
\hline $98 \mathrm{~L}$ & 94333 & 92201 & 711 & 94331 & 92199 & 711 & $\begin{array}{l}\text { Early transcription } \\
\text { factor, VETF1 }\end{array}$ & DPV107 & $78 / 90$ & LSDV098 & $77 / 90$ & WR126 & $71 / 86$ & M96L & $76 / 89$ & $98 \mathrm{~L}$ & $91 / 96$ \\
\hline $99 \mathrm{R}$ & 94386 & 95255 & 290 & 94384 & 95253 & 290 & $\begin{array}{l}\text { Intermediate } \\
\text { transcription factor } \\
\text { VITF-3 }\end{array}$ & DPV108 & $65 / 81$ & LSDV099 & $67 / 82$ & WR127 & $61 / 80$ & M97R & $68 / 83$ & $99 \mathrm{R}$ & $90 / 95$ \\
\hline $100 \mathrm{~L}$ & 95494 & 95258 & 79 & 95492 & 95256 & 79 & $\begin{array}{l}\text { IMV membrane } \\
\text { protein }\end{array}$ & DPV109 & $86 / 91$ & LSDV100 & $75 / 84$ & WR128 & $72 / 81$ & M98L & $78 / 90$ & $100 \mathrm{~L}$ & $91 / 94$ \\
\hline $101 \mathrm{~L}$ & 98203 & 95498 & 902 & 98201 & 95496 & 902 & Core protein $\mathrm{P} 4 \mathrm{a}$ & DPV110 & $69 / 85$ & LSDV101 & $67 / 82$ & WR129 & $54 / 72$ & M99L & $61 / 79$ & $101 \mathrm{~L}$ & $92 / 96$ \\
\hline $102 \mathrm{R}$ & 98218 & 99150 & 311 & 98216 & 99148 & 311 & & DPV111 & $78 / 89$ & LSDV102 & $76 / 89$ & WR130 & $55 / 75$ & M100R & $75 / 88$ & $102 \mathrm{R}$ & $91 / 96$ \\
\hline $103 \mathrm{~L}$ & 99672 & 99166 & 169 & 99670 & 99164 & 169 & Core protein & DPV112 & $58 / 72$ & LSDV103 & $55 / 70$ & WR131 & $46 / 63$ & M101L & $77 / 86$ & $103 \mathrm{~L}$ & $77 / 86$ \\
\hline $104 \mathrm{~L}$ & 99920 & 99717 & 68 & 99918 & 99715 & 68 & $\begin{array}{l}\text { IMV membrane } \\
\text { protein }\end{array}$ & DPV113 & $57 / 75$ & LSDV104 & $56 / 79$ & & & M102L & $50 / 70$ & $104 \mathrm{~L}$ & $83 / 94$ \\
\hline $105 \mathrm{~L}$ & 100248 & 99970 & 93 & 100246 & 99968 & 93 & $\begin{array}{l}\text { IMV } \\
\text { phosphoprotein }\end{array}$ & DPV114 & $86 / 94$ & LSDV105 & $78 / 90$ & WR133 & $61 / 77$ & M103L & $73 / 83$ & $105 \mathrm{~L}$ & $98 / 94$ \\
\hline 106L & 100426 & 100268 & 53 & 100424 & 100266 & 53 & $\begin{array}{l}\text { IMV membrane } \\
\text { virulence factor }\end{array}$ & DPV115 & $84 / 94$ & LSDV106 & $79 / 88$ & WR134 & $66 / 80$ & M104L & $79 / 88$ & $106 \mathrm{~L}$ & $100 / 100$ \\
\hline $107 \mathrm{~L}$ & 100700 & 100419 & 94 & 100698 & 100417 & 94 & & DPV116 & $50 / 69$ & LSDV107 & $52 / 71$ & WR135 & $52 / 67$ & M105L & $51 / 72$ & $107 \mathrm{~L}$ & $78 / 88$ \\
\hline $108 \mathrm{~L}$ & 101829 & 100687 & 381 & 101827 & 100685 & 381 & IMV protein & DPV117 & $64 / 81$ & LSDV108 & $63 / 80$ & WR136 & $51 / 70$ & M106L & $55 / 73$ & $108 \mathrm{~L}$ & $79 / 87$ \\
\hline 109L & 102418 & 101846 & 191 & 102416 & 101844 & 191 & $\begin{array}{l}\text { IMV membrane } \\
\text { phosphoprotein }\end{array}$ & DPV118 & $75 / 90$ & LSDV109 & $61 / 78$ & WR137 & $41 / 63$ & M107L & $57 / 73$ & $109 \mathrm{~L}$ & $92 / 97$ \\
\hline $110 \mathrm{R}$ & 102433 & 103869 & 479 & 102431 & 103867 & 479 & DNA helicase & DPV119 & $62 / 81$ & LSDV110 & $58 / 76$ & WR138 & $58 / 76$ & M108R & $61 / 79$ & $110 \mathrm{R}$ & $85 / 94$ \\
\hline $111 \mathrm{~L}$ & 104077 & 103856 & 74 & 104075 & 103854 & 74 & & DPV120 & $76 / 90$ & LSDV111 & $72 / 87$ & WR139 & $62 / 77$ & M109L & $81 / 90$ & $111 \mathrm{~L}$ & $81 / 94$ \\
\hline $112 \mathrm{~L}$ & 104410 & 104081 & 110 & 104408 & 104079 & 110 & Fusion protein & DPV122 & $57 / 73$ & LSDV113 & $57 / 72$ & WR140 & $57 / 70$ & M110L & $56 / 74$ & $112 \mathrm{~L}$ & $82 / 91$ \\
\hline $113 R$ & 104409 & 105683 & 425 & 104407 & 105681 & 425 & $\begin{array}{l}\text { DNA polymerase } \\
\text { processivity factor }\end{array}$ & DPV121 & $50 / 71$ & LSDV112 & $51 / 66$ & WR141 & $46 / 66$ & M111R & $47 / 66$ & $113 R$ & $76 / 89$ \\
\hline $114 \mathrm{R}$ & 105695 & 106165 & 157 & 105693 & 106163 & 157 & DNA processing & DPV123 & $72 / 88$ & LSDV114 & $67 / 83$ & WR142 & $67 / 86$ & M112R & $63 / 83$ & $114 R$ & $77 / 89$ \\
\hline $115 \mathrm{R}$ & 106190 & 107338 & 383 & 106188 & 107336 & 383 & $\begin{array}{l}\text { Intermediate } \\
\text { transcription factor } \\
\text { VITF-3 }\end{array}$ & DPV124 & $60 / 79$ & LSDV115 & $63 / 78$ & WR143 & $60 / 77$ & M113R & $60 / 76$ & $115 R$ & $84 / 91$ \\
\hline
\end{tabular}




\begin{tabular}{|c|c|c|c|c|c|c|c|c|c|c|c|c|c|c|c|c|c|}
\hline $116 \mathrm{R}$ & 107343 & 110837 & 1165 & 107341 & 110835 & 1165 & $\begin{array}{l}\text { RNA polymerase } \\
\text { subunit rpo132 }\end{array}$ & DPV125 & $88 / 96$ & LSDV116 & $89 / 96$ & WR144 & $82 / 92$ & M114R & $85 / 94$ & $116 \mathrm{R}$ & $94 / 98$ \\
\hline $117 \mathrm{~L}$ & 111283 & 110840 & 148 & 111281 & 110838 & 148 & Fusion protein & DPV126 & $39 / 61$ & LSDV117 & $41 / 64$ & WR150 & $43 / 61$ & M115L & $43 / 66$ & $117 \mathrm{~L}$ & $41 / 64$ \\
\hline $118 \mathrm{~L}$ & 111703 & 111287 & 139 & 111701 & 111285 & 139 & $\begin{array}{l}\text { Viral replication } \\
\text { A28-like }\end{array}$ & DPV127 & $70 / 85$ & LSDV118 & $61 / 78$ & WR151 & $54 / 71$ & M116L & $61 / 80$ & $118 \mathrm{~L}$ & $78 / 92$ \\
\hline 119L & 112618 & 111719 & 300 & 112616 & 111717 & 300 & $\begin{array}{l}\text { RNA polymerase } \\
\text { subunit rpo35 }\end{array}$ & DPV128 & $64 / 79$ & LSDV119 & $66 / 77$ & WR152 & $57 / 76$ & M117L & $61 / 77$ & $119 \mathrm{~L}$ & $85 / 92$ \\
\hline $120 \mathrm{~L}$ & 112814 & 112590 & 75 & 112812 & 112588 & 75 & IMV membrane & DPV129 & $69 / 82$ & LSDV120 & $58 / 77$ & WR153 & $54 / 84$ & M118L & $63 / 75$ & $120 \mathrm{~L}$ & 90/94 \\
\hline $120.5 \mathrm{~L}$ & 112978 & 112847 & 44 & 112976 & 112845 & 44 & & & & & & & & & & $120.5 \mathrm{~L}$ & $69 / 81$ \\
\hline $121 \mathrm{~L}$ & 113777 & 113019 & 253 & 113775 & 113017 & 253 & $\begin{array}{l}\text { GTPase; DNA } \\
\text { packaging }\end{array}$ & DPV131 & $83 / 94$ & LSDV121 & $83 / 92$ & WR155 & $59 / 76$ & M120L & $81 / 92$ & $121 \mathrm{~L}$ & $89 / 96$ \\
\hline $122 \mathrm{R}$ & 113889 & 114446 & 186 & 113887 & 114444 & 186 & EEV glycoprotein & DPV132 & $44 / 59$ & LSDV122 & $41 / 55$ & WR156 & $33 / 57$ & M121R & $39 / 57$ & $122 \mathrm{R}$ & $66 / 79$ \\
\hline $123 \mathrm{R}$ & 114472 & 114981 & 170 & 114470 & 114979 & 170 & $\begin{array}{l}\text { C-type lectin-like } \\
\text { domain; EEV } \\
\text { glycoprotein }\end{array}$ & DPV133 & $64 / 82$ & LSDV123 & $54 / 75$ & WR157 & $48 / 68$ & M122R & $57 / 81$ & $123 \mathrm{R}$ & $79 / 92$ \\
\hline $124 \mathrm{R}$ & 115022 & 115558 & 179 & 115020 & 115556 & 179 & & DPV134 & $44 / 66$ & LSDV124 & $40 / 60$ & WR158 & $40 / 57$ & M123R & $41 / 60$ & $124 \mathrm{R}$ & $72 / 84$ \\
\hline $125 \mathrm{R}$ & 115597 & 116451 & 285 & 115595 & 116449 & 285 & & DPV135 & $38 / 59$ & LSDV125 & $37 / 62$ & & & M124R & $35 / 58$ & $125 \mathrm{R}$ & $81 / 92$ \\
\hline $126 \mathrm{R}$ & 116512 & 117180 & 223 & 116510 & 117178 & 223 & EEV glycoprotein & DPV136 & $34 / 49$ & & & & & & & $126 \mathrm{R}$ & $35 / 54$ \\
\hline $127 \mathrm{R}$ & 117228 & 118037 & 270 & 117226 & 118035 & 270 & & DPV137 & $39 / 61$ & LSDV127 & $36 / 58$ & WR160 & $27 / 51$ & M126R & $35 / 55$ & $127 R$ & $70 / 85$ \\
\hline $128 \mathrm{~L}$ & 118847 & 118038 & 270 & 118839 & 118036 & 268 & CD47-like & DPV139 & $32 / 57$ & LSDV128 & $31 / 52$ & WR162 & $23 / 40$ & M128L & $31 / 54$ & $128 \mathrm{~L}$ & $66 / 83$ \\
\hline $129 \mathrm{R}$ & 118851 & 119264 & 138 & 118843 & 119256 & 138 & Myristylprotein & DPV138 & $50 / 67$ & & & WR063 & $28 / 45$ & M129R & $36 / 55$ & $129 \mathrm{R}$ & $76 / 88$ \\
\hline $130 \mathrm{~L}$ & 119813 & 119256 & 186 & 119805 & 119248 & 186 & & & & & & & & & & & \\
\hline $131 \mathrm{R}$ & 119889 & 120092 & 68 & 119881 & 120084 & 68 & & & & & & & & & & $131 \mathrm{R}$ & $38 / 65$ \\
\hline $132 \mathrm{R}$ & 120123 & 120368 & 82 & 120115 & 120360 & 82 & & DPV141 & $46 / 66$ & LSDV130 & $45 / 68$ & & & & & $132 \mathrm{R}$ & $63 / 80$ \\
\hline $133 \mathrm{~L}$ & 121408 & 120380 & 343 & 121400 & 120372 & 343 & $\begin{array}{l}\text { 3-Beta } \\
\text { hydroxysteroid } \\
\text { dehydrogenase }\end{array}$ & DPV142 & $53 / 69$ & & & WR170 & $43 / 63$ & & & & \\
\hline $134 \mathrm{R}$ & 121447 & 121914 & 158 & 121439 & 121906 & 156 & IL-24-like & & & LSDV005 & $26 / 47$ & & & & & & \\
\hline $135 \mathrm{R}$ & 121993 & 127701 & 1903 & 121985 & 127693 & 1903 & VARV B22R-like & DPV146 & $48 / 65$ & LSDV134 & $48 / 63$ & & & M134R & $43 / 59$ & $135 \mathrm{R}$ & $78 / 89$ \\
\hline $136 \mathrm{R}$ & 127701 & 128753 & 351 & 127693 & 128745 & 351 & $\begin{array}{l}\text { Type-I IFN } \\
\text { receptor }\end{array}$ & DPV147b & $28 / 51$ & LSDV135 & $33 / 53$ & WR200 & $26 / 44$ & M135R & $23 / 41$ & & \\
\hline $137 \mathrm{R}$ & 128783 & 129241 & 153 & 128775 & 129233 & 153 & A52R-family & DPV148 & $37 / 60$ & LSDV136 & $42 / 68$ & WR022 & $22 / 45$ & M136R & $28 / 55$ & $137 \mathrm{R}$ & $72 / 86$ \\
\hline $138 \mathrm{R}$ & 129270 & 130283 & 338 & 129262 & 130275 & 338 & & DVP149 & $43 / 61$ & LSDV137 & $42 / 60$ & WR177 & $35 / 59$ & M137R & $32 / 55$ & $138 \mathrm{R}$ & $64 / 80$ \\
\hline $139 \mathrm{R}$ & 130362 & 130931 & 190 & 130354 & 130923 & 190 & A52R-family & DPV152 & $50 / 69$ & & & WR039 & $26 / 44$ & M139R & $44 / 66$ & $139 \mathrm{R}$ & $68 / 81$ \\
\hline $140 \mathrm{R}$ & 130966 & 132675 & 570 & 130958 & 132667 & 570 & Kelch-like & DPV160 & $27 / 47$ & LSDV151 & $28 / 47$ & WR180 & $31 / 56$ & M140R & $43 / 62$ & & \\
\hline $141 R$ & 132705 & 133064 & 120 & 132697 & 133056 & 120 & CD200-like & DPV153 & $44 / 63$ & LSDV138 & $52 / 69$ & & & M141R & $38 / 60$ & $141 \mathrm{R}$ & $72 / 82$ \\
\hline $142 \mathrm{R}$ & 133101 & 134027 & 309 & 133093 & 134019 & 309 & $\begin{array}{l}\text { Serine/threonine } \\
\text { protein kinase }\end{array}$ & DPV154 & $58 / 76$ & LSDV139 & $60 / 80$ & WR183 & $47 / 64$ & M142R & $56 / 74$ & $142 \mathrm{R}$ & $84 / 92$ \\
\hline $143 R$ & 134063 & 134764 & 234 & 134055 & 134756 & 234 & $\begin{array}{l}\text { Kila-N/RING } \\
\text { finger }\end{array}$ & DPV155 & $38 / 61$ & LSDV140 & $38 / 60$ & WR208 & $25 / 45$ & M143R & $46 / 64$ & $143 R$ & $80 / 91$ \\
\hline $144 R$ & 134798 & 135601 & 268 & 134790 & 135593 & 268 & $\begin{array}{l}\text { vCCP/EEV host } \\
\text { range }\end{array}$ & DPV156 & $42 / 59$ & LSDV141 & $37 / 56$ & WR025 & $33 / 53$ & M144R & $34 / 52$ & $144 \mathrm{R}$ & $64 / 76$ \\
\hline $145 \mathrm{R}$ & 135900 & 136868 & 323 & 135892 & 136860 & 323 & vCCR8 & DPV162 & $36 / 60$ & LSDV011 & $37 / 62$ & & & & & $145 \mathrm{R}$ & $66 / 78$ \\
\hline $146 \mathrm{R}$ & 137021 & 138427 & 469 & 137013 & 138431 & 473 & Ankyrin repeat & DPV164 & $41 / 63$ & LSDV147 & $37 / 59$ & WR186 & $21 / 41$ & M149R & $34 / 56$ & $146 \mathrm{R}$ & $75 / 86$ \\
\hline $147 \mathrm{R}$ & 138465 & 139937 & 491 & 138456 & 139928 & 491 & Ankyrin repeat & DPV164 & $29 / 52$ & LSDV148 & $35 / 54$ & WR186 & $23 / 39$ & M149R & $23 / 58$ & $147 \mathrm{R}$ & $78 / 89$ \\
\hline
\end{tabular}




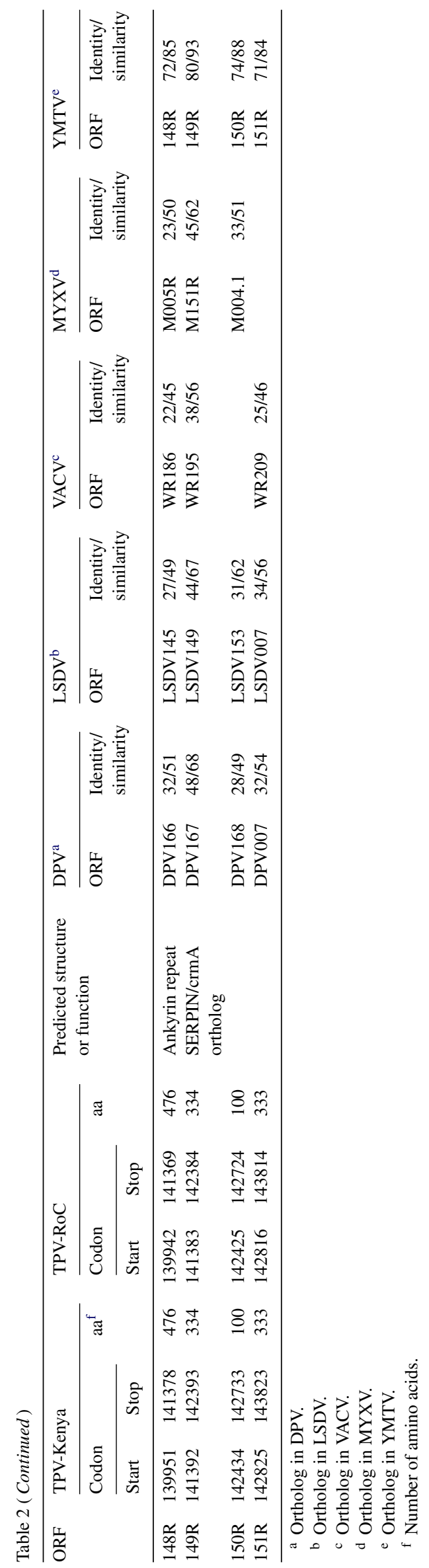

al., 2005). A BLAST search of the intact $11 \mathrm{~L}$ protein revealed homologues only in YLDV (11L), YMTV (11L), Deerpox virus (DPV; DPV019) and Vaccinia virus (VACV; VACV WR186). However, when $11.2 \mathrm{~L}$ was used as the query sequence, additional homologues in Myxoma virus (MYXV; M148R), Lumpy skin disease virus (LSDV; LSDV145) and an additional VACV protein encoded by VACV WR019 were detected. The proteins encoded by TPV11L, DPV019, M148R, LSDV145, WR186 and WR019 range from 558 to 675 amino acids and contain 7-14 predicted ankyrin repeats (Fig. 2A). While all proteins except for VACV WR019 contain the entire predicted F-box domain (Mercer et al., 2005), there is significant sequence similarity outside of the domain (Fig. 2B). It may be that the sequence, found between the last predicted ankyrin repeat and the start of the F-box domain, acts as an important functional determinant of the proteins. The fact that $11 \mathrm{~L}$ is truncated in TPV-Kenya suggests that all 14 ankyrin domains are not required to remain functional. Alternatively, the potential gene products from ORFs 11.1L and 11.2L might interact and form a functional complex.

A previously unidentified ORF was annotated between ORFs 23L and 24L of YMTV and denoted 23.5L (Brunetti et al., 2003). A truncated ortholog was found in YLDV. We find that neither isolate of TPV contains a full-length copy of this predicted ORF, as compared with YMTV. However each isolate encodes for a truncated version of the 23.5L (Fig. 1B). TPV-Kenya encodes a 50 aa ORF that aligns to the carboxy half of YMTV 23.5L and is $98 \%$ identical. In contrast, TPV-RoC encodes a 38 aa ORF which is $71 \%$ identical to the amino terminus of YMTV $23.5 \mathrm{~L}$ (Fig. 1B). A transversion at position 17890 changes a tyrosine (TPV-Kenya) to a stop codon (TPV-RoC) causing premature termination of TPV-RoC 23.5L. As well, an insertion at position 17994 changes a string of thymines from T5 (TPV-RoC) to T6 (TPV-Kenya) and disrupts the coding from the downstream start codon on the minus strand.

\subsection{Overall nucleotide comparative analysis}

Comparison of the two TPV isolates on a nucleotide-bynucleotide basis indicates 35 changes across a pairwise sequence alignment of 144,565 nucleotide positions. Thirty-one of the changes were within predicted coding regions and could be divided into 13 transitions, 12 transversions and 6 deletions. Six transitions cause only synonymous codon changes. The other seven transitions resulted in non-synonymous substitutions within the coding sequence resulting in a single amino acid difference between the comparable protein sequences between the two TPV isolates. Six of these non-synonymous events resulted in relatively non-conserved changes. In contrast, 11 of the 12 transversions were non-synonymous and 10 of the 11 non-synonymous changes were to non-conserved amino acids. An A to $\mathrm{C}$ transversion at position 10241 changes a stop codon (TAG) on the minus strand template of $11.1 \mathrm{~L}$ of TPV-Kenya to a glutamic acid in TPV-RoC resulting in a fulllength $11 \mathrm{~L}$ ORF, comparable in length to the other poxvirus $11 \mathrm{~L}$ orthologs. The 6 deletions represent the absence of one of four hexanucleotide direct repeats (CATATA) present at the 


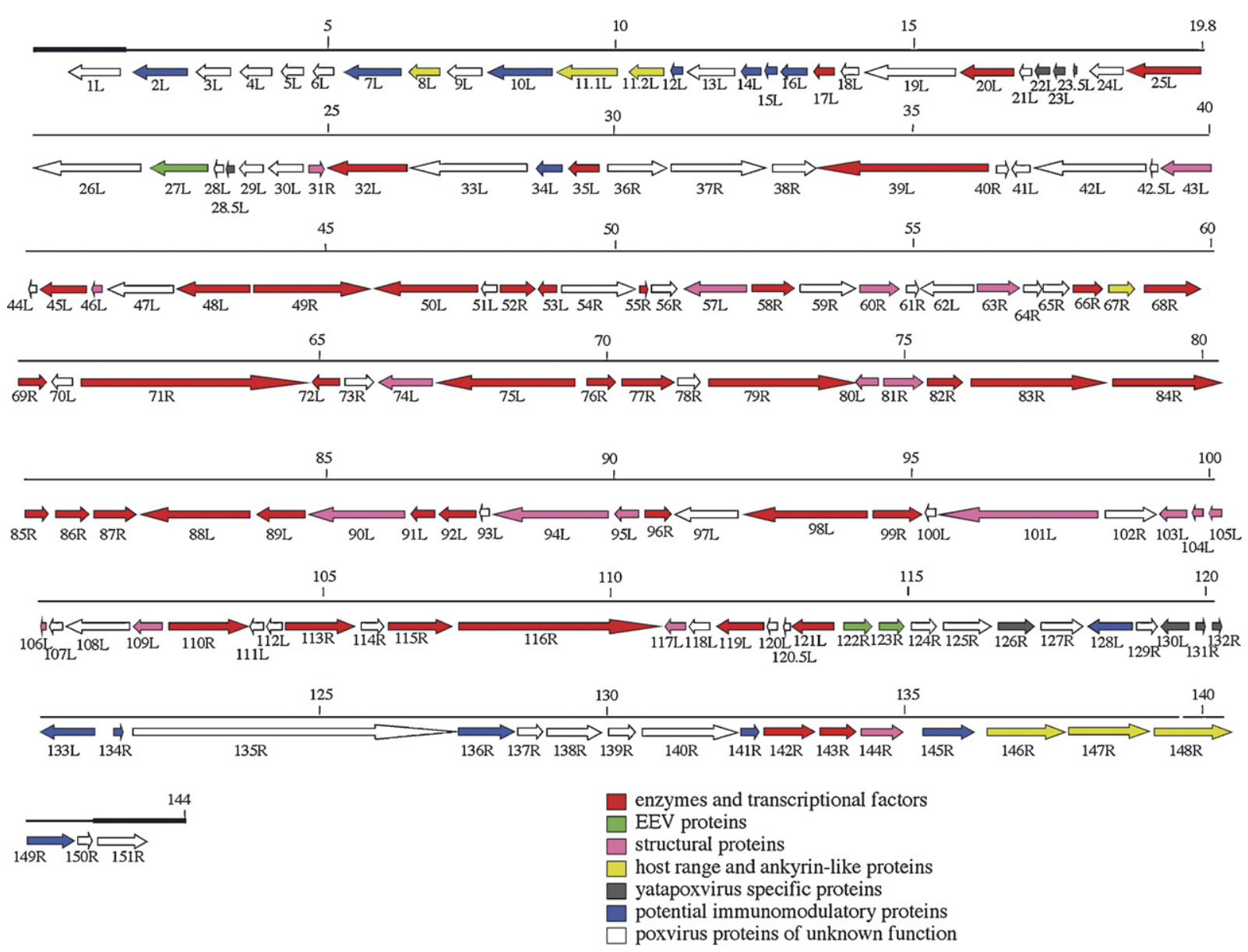

Fig. 3. TPV-Kenya genomic map. ORFs are displayed as arrows that also indicate the direction of transcription. The arrows are coloured to indicate a specific functional category. At either end of the genome is a bolded section that indicates the terminal inverted repeat.

$5^{\prime}$ end of ORF 128L in TPV-RoC. The result of this hexanucleotide deletion results in a shortened $128 \mathrm{~L}$ amino acid sequence in TPV-RoC (MYMYMYNY) compared to TPVKenya (MYMYMYMYNY). The other sequence differences that distinguish the TPV isolates include two transitions in non-coding sequences and an insertion in the intergenic region between ORFs $23 \mathrm{~L}$ and $23.5 \mathrm{~L}$ of a thymidine $(\mathrm{T})$ repeat; $\mathrm{T} 8$ in TPV-RoC compared to T7 in TPV-Kenya.

\subsection{Conserved DNA sequence near the termini}

While examining the intergenic regions of TPV, a predicted 58-codon ORF was found within the TIR, located between the extreme terminus and $1 \mathrm{~L} / 151 \mathrm{R}$. The ORF is transcribed toward the center of the genome and in the opposite direction of all ORFs $20 \mathrm{kbp}$ from either end of the DNA (Fig. 3). The ORF is also present in the YLDV sequence in GenBank but was not described in the publication, possibly due to the ORF mirrorimage orientation, which might contribute to dsRNA production (Lee et al., 2001). The comparable region in YMTV was previously described as a pseudogene (Brunetti et al., 2003). To determine the likelihood that the ORF encodes a functional protein, a translated BLAST search (tBLASTx) was used to find homologous amino acid sequences. Several poxviruses, includ- ing YLDV, YMTV, LSDV and DPV, have sequences that show significant homology to the TPV query sequence; however, the nucleic acid sequences in YMTV, LSDV and DPV lacked a start codon. Therefore, the cognate sequences appear to represent either a pseudogene or a terminal DNA sequence conserved across genera. The region of nucleotide conservation consists of a 300 nucleotide segment that surrounds the predicted 58codon ORF. The TIR regions of all chordopoxviruses were compared for similarity to these conserved sequences. The 300

Table 3

TIR conserved sequence positions in various poxvirus species

\begin{tabular}{lcccll}
\hline Virus & \multicolumn{2}{l}{ Left end } & & \multicolumn{2}{l}{ Right end } \\
\cline { 2 - 3 } & Start & End & & Start & End \\
\hline TPV-Kenya & 400 & 714 & & 144164 & 143850 \\
TPV-RoC & 400 & 714 & & 144154 & 143840 \\
YLDV & 418 & 732 & & 144158 & 143844 \\
YMTV & 437 & 751 & & 134285 & 133971 \\
GTPV & 1018 & 1329 & & 148582 & 148271 \\
LSDV & 1286 & 1592 & & 149488 & 149182 \\
SHPV & 1225 & 1530 & & 148833 & 148528 \\
SWPV & 1062 & 1381 & & 145393 & 145074 \\
DPV & 4756 & 5070 & & 165805 & 165491 \\
\hline
\end{tabular}


nucleotide conserved sequence was found in Swinepox virus (SWPV) and overlapped with a hypothetical gene (designated 002) in SWPV, DPV and Capripoxviruses (Table 3). While this sequence exhibits homology across genera, the highest identities were found between members within a particular genus (Fig. 4).

A highly conserved sequence, present in the TIR region of orthopoxviruses, has been described previously (Shchelkunov et al., 1998). However, the sequence appears to be distinct from the 300-bp Yatapoxvirus sequences and their cognates (Fig. 4). Since new sequence information has become available from the time that this DNA region was last compared and reported (Baroudy et al., 1982; Shchelkunov et al., 1998), selected orthopoxvirus sequences from CPXV, VACV, VARV, HSPV, TATV, ETCV, MPXV, CMLV and RCNV (acronyms described in Table 1; data not shown) were aligned and compared. Interestingly, CMLV lacks this entire sequence and RCNV encodes for only 137 nucleotides of the $\sim 300$-bp conserved sequences. As previously described, these orthopoxvirus nucleotide sequences are highly homologous, sharing $87-100 \%$ sequence identity ((Shchelkunov et al., 1998) and data not shown).

\subsection{Identification of two conserved poxvirus gene families}

Two TPV intergenic regions contained potential ORFs below the commonly used codon limit of 50. The ORFs are located between $27 \mathrm{~L}$ and $28.5 \mathrm{~L}$, and $42 \mathrm{~L}$ and $43 \mathrm{~L}$; they were designated $28 \mathrm{~L}$ and $42.5 \mathrm{~L}$, respectively (Table 2 ). To determine if these ORFs likely encode functional proteins, tBLASTx was used to find homologous sequences. However, due to the small

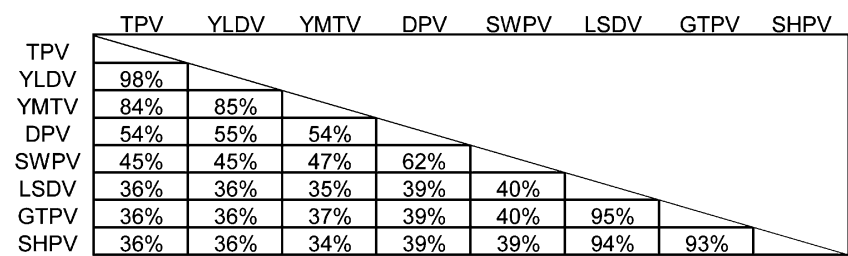

Fig. 4. Identity matrix of a conserved sequence found in the TIR of various poxvirus species. An approximately $300 \mathrm{bp}$ region within the TIR of the poxviruses listed was aligned using ClustalW and percent identity was determined. Poxviruses are listed in order of relatedness for this particular sequence.

size of these ORFs, BLAST searches were unable to detect any homologous sequences and thus the search for homologues was performed manually.

The ORF 28L is present in both TPV and YLDV; the predicted ORF encodes for a potential protein of 48 aa. The region between orthologues of $27 \mathrm{~L}$ and $28.5 \mathrm{~L}$ of the genomes of species of chordopoxviruses currently available were examined. Orthologues were identified in all orthopoxviruses, parapoxviruses, SWPV, and the unclassified poxviruses DPV and Crocodilepox virus (CRV) (Fig. 5a). However, the closely related capripoxviruses lacked a homologous gene in this region. Members of the genera Avipoxvirus, Leporipoxvirus and Molluscum contagiosum virus also lacked the sequence (Table 4).

The ORF $42.5 \mathrm{~L}$ is predicted to encode a 30 amino acid protein. A search of all poxvirus genomes for orthologues showed that ORF $42.5 \mathrm{~L}$ is highly conserved among the Chordopoxvirinae and orthologues in all vertebrate poxviruses currently

(A)

\begin{tabular}{|c|c|c|c|c|c|c|c|c|c|c|c|c|c|c|c|c|c|c|c|c|c|c|c|c|c|c|c|c|c|c|c|c|c|c|c|c|c|c|c|c|c|}
\hline & & & & & & & & & 10 & & & & & & & & & & & & & & & & & & & 30 & & & & & & & & & & & & & \\
\hline TPV28L & M & $\mathrm{E}$ & A & p & $N$ & $\mathrm{~N} N$ & $\mathrm{~s}$ & $\mathrm{~L}$ & $\bar{Y}$ & L & $\mathbf{E}$ & $\bar{G}$ & $\mathrm{~V}$ & $\mathrm{~K}$ & & - & & - & L & $\sqrt{5}$ & & n & $F$ & & & - & - & - & - & - & - & - & L & L & L & L & & & & $7 \mathrm{~N}$ & \\
\hline YLDV28L & $M$ & E & A & p & $P$ & $\mathrm{~N}$ & $\mathrm{~s}$ & L & $Y$ & L & $\mathbf{E}$ & G & v & $\mathrm{K}$ & I & - & - & - & L & 5 & & & 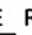 & & & . & - & - & - & - & - & - & L & L & L & L & $F$ & & 11 & $N$ & $\mathrm{~T}$ \\
\hline DPV036 & $M$ & 1 & S & 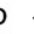 & - & - & - & I & Y & $\mathrm{T}$ & E & G & L & $\mathrm{R}$ & I & $S$ & $\mathrm{~N}$ & E & L & $k$ & & - & $7 c$ & $c$ & & . & - & - & - & - & - & D & D & I & L & $\mathrm{N}$ & M & & $Y D$ & A & V \\
\hline SWPV026 & $M$ & $\mathrm{~F} L$ & $s$ & & - & - & - & v & Y & K & $\mathbf{E}$ & G & 1 & E & L & C & K & $\mathrm{E}$ & $E$ & 1 & & - & $-1 c$ & & & . & 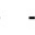 & - & Y & E & $\mathrm{F}$ & E & $Y$ & V & $S$ & D & D & & $\mathrm{ND}$ & A & I \\
\hline EVM037 & $M$ & K I & $\mathrm{H}$ & & - & - & - & v & Y & S & E & G & L & D & I & $S$ & T & D & 17 & $\sqrt{\mathrm{s}}$ & & 1 & 15 & ; & & 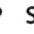 & A & L & D & - & - & M & $Y$ & M & D & $\mathrm{E}$ & D & & IV & $\mathrm{E}$ & L \\
\hline WR053 & $M$ & K & H & & - & - & & L & $Y$ & $\mathrm{~s}$ & E & G & L & S & I & $\mathrm{s}$ & $\mathrm{N}$ & $D$ & 1 & $\mathrm{v}$ & & I & $c$ & $c$ & & $\mathrm{~s}$ & $T$ & M & D & T & D & 1 & $E$ & 1 & D & $E$ & D & & I $M$ & $E$ & 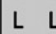 \\
\hline ORF012.5 & $M$ & $S$ & - & - & - & - & - & - & V & L & L & G & L & $S$ & I & $S$ & - & - & A & 4 & & $v$ & $F$ & ; & & 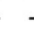 & - & - & - & - & - & V & L & G & L & C & G & & $\mathrm{G}$ L & E & 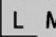 \\
\hline CRV044 & $M$ & $s$ & $S$ & & $P \quad P$ & o & & $\mathrm{V}$ & $\mathrm{s}$ & $\mathrm{P}$ & $\mathrm{T}$ & $\mathrm{G}$ & $N$ & v & L & $E$ & D & G & s & 5 & & F & 日 & 11 & & a. & - & - & - & - & I & L & & & c & 1 & v & & & & \\
\hline & & & & 5 & 0 & & & & & & & & & 60 & & & & & & & & & 70 & & & & & & & & & & 80 & & & & & & & & \\
\hline PV28L & $\mathrm{N}$ & $\mathrm{R}$ & $\bar{L}$ & 1 & $K \longdiv { L }$ & - & - & - & - & - & - & - & - & & $\bar{F}$ & $\mathrm{~N}$ & - & - & $-\Gamma$ & $7 \mathrm{k}$ & & N & $k N$ & 1 & & & & & & & & & & & & & & & & & \\
\hline YLDV28L & N & $R$ & L & v & $\mathrm{K}$ L & - & - & - & - & - & - & - & - & $\mathrm{N}$ & $F$ & $\mathrm{~N}$ & - & - & - & k & & v & $k 1$ & & & & & & & & & & & & & & & & & & \\
\hline DPV036 & $\mathrm{N}$ & $T$ & I & 5 & I & I & v & L & 1 & D & L & L & E & $\mathrm{N}$ & $M$ & $Y$ & L & - & - & 10 & 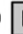 & D & - & 11 & & E & A & - & - & - & - & $\mathrm{E}$ & L & Q & Q & $\mathrm{L}$ & S & T & D & & \\
\hline SWPV026 & $D$ & M & I & v & $\mathrm{T} \mathrm{L}$ & 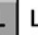 & $\mathrm{N}$ & - & - & - & - & - & - & $E$ & $\bar{F}$ & E & T & - & - & 10 & 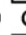 & $E$ & 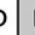 & 71 & & 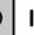 & $\mathrm{N}$ & - & - & - & - & $\mathrm{N}$ & $\mathrm{N}$ & $E$ & $\mathrm{E}$ & v & & & & & \\
\hline EVM037 & $\mathrm{N}$ & 1 & L & $\mathrm{r}$ & $\bar{E}$ & $G$ & C & D & V & D & $\mathrm{F}$ & D & $\mathrm{E}$ & $\mathrm{N}$ & $\mathbf{F}$ & $\mathrm{S}$ & D & - & - & A & & ) & 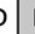 & & & 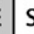 & L & - & - & - & - & V & $\mathrm{E}$ & Q & D & v & & & & & \\
\hline WR053 & $\mathrm{N}$ & 1 & L $\quad T$ & r & $E$ L & -6 & c & D & v & D & $\mathrm{F}$ & D & $E$ & $\mathrm{~N}$ & $\mathbf{F}$ & $\mathrm{S}$ & D & - & - & A & & & 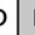 & & & $\mathrm{s}$ & L & - & - & - & - & I & E & Q & D & v & & & & & \\
\hline ORF012.5 & $\mathrm{v}$ & $\mathrm{L}$ & $\mathrm{F}$ & 4 & $E R$ & $R$ & v & A & A & $\mathbf{R}$ & - & - & - & $R$ & $\mathrm{Q}$ & & E & - & - & 10 & & E & 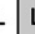 & -1 & & c & & Q & - & - & - & $\mathrm{E}$ & G & $\mathrm{E}$ & $\mathrm{F}$ & v & N & Y & & & \\
\hline RV044 & c & L & $L$ & 1 & E L & $1 \mathrm{v}$ & $Y$ & 11 & I & $\mathrm{R}$ & - & K & $\mathrm{R}$ & & $F$ & H & c & $P$ & I & 5 & & E & $\mathrm{k}$ & & & $P$ & & $S$ & S & $\mathrm{P}$ & $P$ & & & $\vec{A}$ & & 1 & & & D & & \\
\hline & & & & & & & & & 1 & 0 & & & & & & & & & 20 & & & & & & & & & & 0 & & & & & & & & & 40 & & & \\
\hline TPV42.5L & $\sqrt{M}$ & 11 & & 1 & $\mathrm{v}$ & 1 & $F$ & $\mathrm{~L} \Gamma$ & I & $\bar{A}$ & $\mathrm{~F}$ & $s[$ & $F C$ & & $\sqrt{\mathrm{V}}$ & $\sqrt{12}$ & .5 & $\mathrm{Y}$ & L & $F$ & L & K & $P$ & $Y$ & 1 & v & I & G & A & & & & & & & & & & & & \\
\hline YLDV42.5L & $M$ & 11 & & I & v & 1 & $\mathbf{F}$ & L & 11 & A & $\mathbf{F}$ & $s$ & $F C$ & & $\Lambda \mid v$ & 1 & - 5 & $Y$ & L & $\mathbf{F}$ & L & K & $\mathbf{P}$ & Y & I & v & 1 & G & A & & & & & & & & & & & & \\
\hline YMTV42.5L & M & 11 & $\mathbf{v}$ & $\mathrm{F}$ & $\mathrm{T}$ & 1 & $\mathbf{F}$ & 1 & 11 & A & $F$ & G & $F C$ & & V & 1 & $s$ & $Y$ & L & $S$ & L & K & $\mathbf{P}$ & $Y$ & V & v & 1 & $\mathrm{~T}$ & & & & & & & & & & & & & \\
\hline LSDV042.5 & M & 11 & $\mathbf{v}$ & L & $\mathbf{v}$ & $F$ & $\mathbf{F}$ & $\mathrm{F}$ & 1 & A & $\mathbf{F}$ & s & F C & & $v v$ & $\square$ & Ds & $Y$ & $\mathrm{~s}$ & $F$ & L & $K$ & $\mathbf{P}$ & $Y$ & $T$ & $Y$ & K & I & & & & & & & & & & & & & \\
\hline DPV050.5 & M & 11 & $\mathbf{v}$ & 1 & $\mathbf{v}$ & $M$ & $\mathbf{F}$ & $\mathrm{F}$ & & A & $\mathbf{F}$ & c & F C & & I V & $v 1$ & s & $Y$ & $T$ & $\mathbf{F}$ & L & $\mathrm{Y}$ & $\mathbf{P}$ & $Y$ & 1 & $F$ & $F$ & K & $\mathbf{N}$ & K & $D$ & & & 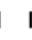 & & & & $\mathrm{H}$ & $\mathbf{R}$ & & $=\mathrm{N}$ \\
\hline M37L & M & 11 & $v$ & $F$ & $\mathbf{v}$ & 1 & $\mathbf{F}$ & 1 & 11 & A & $\mathbf{F}$ & v & $F C$ & E & i & $v$ & ]s & $Y$ & G & $\mathbf{F}$ & L & $K$ & $\mathbf{P}$ & $Y$ & $M$ & $F$ & L & $N$ & $\mathbf{R}$ & K & $H$ & & & & & & & & & & \\
\hline VARV058.5 & M & & v & v & 1 & $M$ & $\mathbf{F}$ & $\mathrm{F}$ & & A & $F$ & A & FC & & & $\sqrt{1}$ & s & $Y$ & 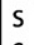 & $Y$ & L & $R$ & $P$ & Y & $T$ & $S$ & $T$ & K & $E$ & L & & & & & & & & & & & \\
\hline VR220 & $M$ & & v & & 1 & M & $\mathbf{F}$ & $F$ & & A & & & & & & & $s$ & $Y$ & $s$ & & & & $P$ & Y & & $S$ & $T$ & K & $\mathrm{E}$ & & & & & & & & & & & & \\
\hline
\end{tabular}

Fig. 5. Alignments of the predicted $28 \mathrm{~L}$ and $42.5 \mathrm{~L}$ gene families. (a) Orthologs of TPV 28L are aligned and include YLDV, DPV, SWPV, ECTV (EVM037), VACV (WR053), ORFV, CRV. (b) Orthologs of TPV 42.5L are aligned and include YLDV, YMTV, LSDV, DPV, MYXV (M37L), VARV, VACV (WR220), as annotated at www.poxvirus.org. 
Table 4

Members of two new poxvirus gene families

\begin{tabular}{|c|c|c|c|c|c|c|c|}
\hline \multirow[t]{2}{*}{ Genus } & \multirow[t]{2}{*}{ Virus } & \multicolumn{3}{|c|}{ 28L gene family } & \multicolumn{3}{|c|}{ 42.5L gene family } \\
\hline & & Designation & Start & Stop & Designation & Start & Stop \\
\hline \multirow{4}{*}{ Yatapox } & TPV-RoC & $28 \mathrm{~L}$ & 23330 & 23187 & $42.5 \mathrm{~L}$ & 39411 & 39322 \\
\hline & TPV-Kenya & $28 \mathrm{~L}$ & 23332 & 23189 & $42.5 \mathrm{~L}$ & 39413 & 39324 \\
\hline & YLDV & $28 \mathrm{~L}$ & 23342 & 23199 & $42.5 \mathrm{~L}$ & 39425 & 39336 \\
\hline & YMTV & $\mathrm{NP}^{\mathrm{a}}$ & & & $42.5 \mathrm{~L}$ & 33938 & 33852 \\
\hline \multirow{3}{*}{ Capripox } & GTPV & NP & & & 038.5 & 37590 & 37504 \\
\hline & SHPV & NP & & & 038.5 & 37805 & 37719 \\
\hline & LSDV & NP & & & 042.5 & 38189 & 38103 \\
\hline Suipox & SWPV & 026 & 19915 & 19724 & 038.5 & 34712 & 34620 \\
\hline \multirow{2}{*}{ Leporipox } & MYXV & NP & & & $37 \mathrm{~L}$ & 39385 & 39290 \\
\hline & SHFV & NP & & & $37 \mathrm{~L}$ & 39399 & 39316 \\
\hline Molluscipox & MOCV & NP & & & $043.5 \mathrm{~L}$ & 64055 & 63933 \\
\hline \multirow{8}{*}{ Orthopox } & ECTV & 037 & 50964 & 50749 & 053.5 & 68659 & 68555 \\
\hline & MPXV & $\mathrm{C} 20 \mathrm{~L}$ & 42461 & 42240 & $\mathrm{I} 0.5 \mathrm{~L}$ & 59903 & 59799 \\
\hline & CPXV & 062 & 58869 & 58648 & 079.5 & 76579 & 76475 \\
\hline & VARV & 041 & 33516 & 33295 & 058.5 & 51129 & 51025 \\
\hline & HSPV & 054 & 53482 & 53261 & 070.5 & 71114 & 71010 \\
\hline & TATV & 054 & 42022 & 41807 & 072.5 & 59706 & 59602 \\
\hline & CMPV & $49 \mathrm{~L}$ & 43284 & 43063 & $66.5 \mathrm{~L}$ & 60901 & 60797 \\
\hline & VACV & 053 & 42188 & 41967 & $220^{\mathrm{b}}$ & 59851 & 59744 \\
\hline \multirow{2}{*}{ Avipox } & FWPV & NP & & & 090.5 & 90681 & 90782 \\
\hline & CNPV & NP & & & 117.5 & 120153 & 120254 \\
\hline \multirow{2}{*}{ Parapox } & ORFV & $012.5^{\mathrm{b}}$ & 11760 & 11578 & 029.5 & 32396 & 32244 \\
\hline & BPSV & $011.5^{\mathrm{b}}$ & 12820 & 12650 & 028.5 & 33354 & 33214 \\
\hline \multirow{2}{*}{ Unclassified } & CRV & 044 & 58267 & 58025 & 064.5 & 86842 & 86762 \\
\hline & DPV & 036 & 32435 & 32226 & 050.5 & 49191 & 49060 \\
\hline
\end{tabular}

a Not present.

b Annotated at www.poxvirus.org/.

sequenced were found (Fig. 5b). The nucleotide sequence has previously only been reported as a putative ORF of 32 codons for sequenced leporipoxviruses (Cameron et al., 1999; Willer et al., 1999). Additionally, an orthologue, VACV ORF WR220, has been annotated in sequences at http://www.poxvirus.org/ (Table 4).

\section{Discussion}

The virtually complete genome sequences were determined for two isolates of TPV recovered from clinical cases that occurred about 50 years apart. Annotation of the determined sequences revealed a single ORF difference between the two genomes. The ORF $11 \mathrm{~L}$ is truncated in TPV-Kenya compared to TPV-RoC, which suggests that even the small genetic variability present between the two genomes has possibly resulted in changes to the proteome. A TPV nucleotide sequence is conserved in the TIR region of several poxviruses closely related to the yatapoxviruses. An analogous but distinct DNA sequence located in the correlate region of the genome is also present in the orthopoxviruses. Finally, two novel gene families are proposed following identification using comparative genomics.

One of the difficulties that arise when limited sequences are available for comparison is that, ORFs that do not meet the standard search parameters can be difficult to assign. Many poxvirus ORFs are quite small and it is unlikely that they will achieve a significant match using BLAST. Selected available sequences from chordopoxviruses were used to determine significantly conserved sequences in TPV. One approach was to compare a tentatively designated ORF and examine areas of several poxviruses that contained highly conserved ORFs flanking this region.

Using this method, two previously unidentified gene families, $28 \mathrm{~L}$ and $42.5 \mathrm{~L}$, which are clearly present in members of several other poxvirus genera, were identified. The region between ORFs $27 \mathrm{~L}$ and $28.5 \mathrm{~L}$ was previously assigned to a large but overlapping ORF (28R) that was identified in YLDV (Lee et al., 2001); therefore ORF 28L was not originally identified as a putative gene. However, the evidence that orthologs of $28 \mathrm{~L}$ are encoded by a variety of poxviruses suggests that $28 \mathrm{~L}$ encodes a protein product (Table 4 ). Conversely, $42.5 \mathrm{~L}$ had previously only been identified in the leporipoxviruses but this ortholog is highly conserved among the Chordopoxvirinae. Due to its extremely small size (30-44 codons), it is unlikely to have been considered an ORF previously. On close inspection, however, $42.5 \mathrm{~L}$ has a conserved early and late promoter (data not shown; www.poxvirus.org/) and the putative amino acid sequence shares $62-77 \%$ identity with orthologs among other chordopoxviruses. 
These properties should be sufficient to designate $42.5 \mathrm{~L}$ as a putative ORF. Other related predictive methods, such as analyzing purine skew of the ORFs (Da Silva and Upton, 2005), were not used since both of these ORFs had clear orthologues in other poxviruses.

In addition to identifying new putative ORFs, a conserved DNA sequence in the TIR of several genera of poxviruses was described. An analogous sequence that exhibited a similar organization pattern, but did not show significant homology, has been identified previously in the orthopoxviruses. A possible role in DNA replication for the orthopoxvirus conserved sequence in the TIR region has been proposed (Shchelkunov et al., 1998), but since there is considerable divergence of the sequence across genera, a precise mechanism is unclear and may be structural rather than sequence-specific. Structural elements such as Holliday Junctions and cruciform structures have been shown to be important for resolution of concatenated DNA into unit length DNA molecules (Palaniyar et al., 1999). This sequence may fulfill its function in a similar way, relying on a structural motif.

If the conserved sequence does, in fact, play a role in DNA replication, then a sequence that performs a similar function must be present in all poxviruses. Therefore, an attempt to define this sequence in other poxvirus species was made. It is possible to find sequences in other poxvirus TIRs that share some homology to the conserved sequence; however, without more sequence information from viral members within genera it is difficult to clearly define since there is a lack of sequence information for viral members within other poxvirus genera, including two genera composed of only a single member each (Suipoxvirus and Molluscipoxvirus).

Through a comparative genomics approach, we have identified important additional features of yatapoxviruses noted by prior sequencing of YMTV and YLDV. The results presented indicate a relatively slow evolutionary rate, which suggests a relatively stable, confined evolutionary niche. From this standpoint, the primate host-range of TPV and YLDV in the central region of the rainforest of Africa appears to have remained the same, at least for 50 years, despite extensive ecological changes, particularly urbanization of forested areas. There have been suggestions that an insect vector might be involved in Yatapoxvirus transmission because TPV and YLDV infection are localized to one or two lesions and not systemic like smallpox (Damon, 2007). Maintaining a lifecycle that includes a potential non-human primate reservoir, an insect reservoir, as well as a human reservoir suggests that a constant genetic selective pressure might be maintained on the TPV and YLDV genome, which would lower the likelihood of sequence divergence. However, this may not explain the lack of nucleotide changes in the third bp position and it is unlikely that the DNA polymerase encoded by Yatapoxviruses has a high enough fidelity to explain this phenomenon. The codon bias present in many Yatapoxvirus genes represent the most rarely used codons in mammalian cells (Barrett et al., 2006). An alternative explanation to explain the third position conservation is that this codon bias is required for efficient gene expression in a variety of distinct host species. In contrast, a poxvirus that is able to infect several different hosts, e.g. Cowpox virus, which appears to be parental to the orthopoxviruses, has a sequence that is more amenable to changing with different hosts.

\section{Acknowledgements}

This work was supported by the Canadian Institutes of Health Research (CIHR) and National Cancer Institute of Canada (NCIC). SN was supported by an Ontario Graduate Scholarship and Western Graduate Research Scholarship. GM held a Canada Research Chair in Molecular Virology and is an International Scholar of the Howard Hughes Medical Institute.

\section{References}

Afonso, C.L., Delhon, G., Tulman, E.R., Lu, Z., Zsak, A., Becerra, V.M., Zsak, L., Kutish, G.F., Rock, D.L., 2005. Genome of deerpox virus. J. Virol. 79 (2), 966-977.

Afonso, C.L., Tulman, E.R., Delhon, G., Lu, Z., Viljoen, G.J., Wallace, D.B., Kutish, G.F., Rock, D.L., 2006. Genome of crocodilepox virus. J. Virol. 80 (10), 4978-4991.

Afonso, C.L., Tulman, E.R., Lu, Z., Zsak, L., Kutish, G.F., Rock, D.L., 2000 The genome of Fowlpox virus. J. Virol. 74 (8), 3815-3831.

Afonso, C.L., Tulman, E.R., Lu, Z., Zsak, L., Osorio, F.A., Balinsky, C., Kutish, G.F., Rock, D.L., 2002. The genome of swinepox virus. J. Virol. 76 (2), 783-790.

Balbas, P., Gosset, G., 2001. Chromosomal editing in Escherichia coli. Vectors for DNA integration and excision. Mol. Biotechnol. 19 (1), 1-12.

Baroudy, B.M., Venkatesan, S., Moss, B., 1982. Incompletely base-paired flipflop terminal loops link the two DNA strands of the vaccinia virus genome into one uninterrupted polynucleotide chain. Cell 28 (2), 315-324.

Barrett, J.W., Sun, Y., Nazarian, S.H., Belsito, T.A., Brunetti, C.R., McFadden, G., 2006. Optimization of codon usage of poxvirus genes allows for improved transient expression in mammalian cells. Virus Genes 33 (1), 15-26.

Brunetti, C.R., Amano, H., Ueda, Y., Qin, J., Miyamura, T., Suzuki, T., Li, X., Barrett, J.W., McFadden, G., 2003. Complete genomic sequence and comparative analysis of the tumorigenic poxvirus Yaba monkey tumor virus. J. Virol. 77 (24), 13335-13347.

Buller, R.M., Arif, B.M., Black, D.N., Dumbell, K.R., Esposito, J.J., Lefkowitz, E.J., McFadden, G., Moss, B., Mercer, A.A., Moyer, R.W., Skinner, M.A., Tripathy, D.N., 2005. Poxviridae. In: Fauquet, C., Mayo, M.A., Maniloff, J., Desselberger, U., Ball, L.A. (Eds.), Virus Taxonomy: Classification and Nomenclature of Viruses; Eighth Report of the International Committee on Taxonomy of Viruses. Elsevier/Academic Press, Oxford, pp. 117-133.

Cameron, C., Hota-Mitchell, S., Chen, L., Barrett, J., Cao, J.-X., Macaulay, C., Willer, D., Evans, D., McFadden, G., 1999. The complete DNA sequence of myxoma virus. Virology 264 (2), 298-318.

Da Silva, M., Upton, C., 2005. Using purine skews to predict genes in AT-rich poxviruses. BMC Genomics 6 (1), 22.

Damon, I.K., 2007. Poxviruses. In: Knipe, D.M., Howley, P.M. (Eds.), Fields Virology, Vol.2, 5th ed. Lippincott, Williams \& Wilkins, New York, pp. 2947-2976.

Delhon, G., Tulman, E.R., Afonso, C.L., Lu, Z., de la Concha-Bermejillo, A., Lehmkuhl, H.D., Piccone, M.E., Kutish, G.F., Rock, D.L., 2004. Genomes of the parapoxviruses ORF virus and bovine papular stomatitis virus. J. Virol. 78 (1), 168-177.

Dhar, A.D., Werchniak, A.E., Li, Y., Brennick, J.B., Goldsmith, C.S., Kline, R., Damon, I., Klaus, S.N., 2004. Tanapox infection in a college student. N. Engl. J. Med. 350 (4), 361-366.

Domi, A., Moss, B., 2002. Cloning the vaccinia virus genome as a bacterial artificial chromosome in Escherichia coli and recovery of infectious virus in mammalian cells. Proc. Natl. Acad. Sci. U.S.A. 99 (19), 12415-12420.

Downie, A.W., Espana, C., 1972. Comparison of Tanapox virus and Yaba-like viruses causing epidemic disease in monkeys. J. Hyg. (Lond.) 70 (1), 23-32. 
Espana, C., Brayton, M.A., Ruebner, B.H., 1971. Electron microscopy of the Tana poxvirus. Exp. Mol. Pathol. 15 (1), 34-42.

Esposito, J.J., Sammons, S.A., Frace, A.M., Osborne, J.D., Olsen-Rasmussen, M., Zhang, M., Govil, D., Damon, I.K., Kline, R., Laker, M., Li, Y., Smith, G.L., Meyer, H., Leduc, J.W., Wohlhueter, R.M., 2006. Genome sequence diversity and clues to the evolution of variola (smallpox) virus. Science 313 (5788), 807-812.

Gubser, C., Smith, G.L., 2002. The sequence of camelpox virus shows it is most closely related to variola virus, the cause of smallpox. J. Gen. Virol. $83(\mathrm{Pt}$ 4), 855-872.

Knight, J.C., Novembre, F.J., Brown, D.R., Goldsmith, C.S., Esposito, J.J., 1989. Studies on Tanapox virus. Virology 172 (1), 116-124.

Lee, H.J., Essani, K., Smith, G.L., 2001. The genome sequence of Yaba-like disease virus, a yatapoxvirus. Virology 281 (2), 170-192.

McNulty Jr., W.P., Lobitz Jr., W.C., Hu, F., Maruffo, C.A., Hall, A.S., 1968. A pox disease in monkeys transmitted to man. Clinical and histological features. Arch. Dermatol. 97 (3), 286-293.

Mercer, A.A., Fleming, S.B., Ueda, N., 2005. F-box-like domains are present in most poxvirus ankyrin repeat proteins. Virus Genes 31 (2), 127-133.

Mercer, A.A., Ueda, N., Friederichs, S.M., Hofmann, K., Fraser, K.M., Bateman, T., Fleming, S.B., 2006. Comparative analysis of genome sequences of three isolates of Orf virus reveals unexpected sequence variation. Virus Res. 116 (1-2), 146-158.

Moss, B., 2007. Poxviridae: the viruses and their replication. In: Knipe, D.M., Howley, P.M. (Eds.), Fields Virology, vol. 2, fifth ed. Lippincott, Williams \& Wilkins, New York, pp. 2905-2946.

Palaniyar, N., Gerasimopoulos, E., Evans, D.H., 1999. Shope fibroma virus DNA topoisomerase catalyses holliday junction resolution and hairpin formation in vitro. J. Mol. Biol. 287 (1), 9-20.
Seet, B.T., Johnston, J.B., Brunetti, C.R., Barrett, J.W., Everett, H., Cameron, C., Sypula, J., Nazarian, S.H., Lucas, A., McFadden, G., 2003. Poxviruses and immune evasion. Annu. Rev. Immunol. 21, 377-423.

Senkevich, T.G., Bugert, J.J., Sisler, J.R., Koonin, E.V., Darai, G., Moss, B., 1996. Genome sequence of a human tumorigenic poxvirus: prediction of specific host response-evasion genes. Science 273, 813-816.

Shchelkunov, S.N., Safronov, P.F., Totmenin, A.V., Petrov, N.A., Ryazankina, O.I., Gutorov, V.V., Kotwal, G.J., 1998. The genomic sequence analysis of the left and right species-specific terminal region of a cowpox virus strain reveals unique sequences and a cluster of intact ORFs for immunomodulatory and host range proteins. Virology 243 (2), 432-460.

Shchelkunov, S.N., Totmenin, A.V., Babkin, I.V., Safronov, P.F., Ryazankina, O.I., Petrov, N.A., Gutorov, V.V., Uvarova, E.A., Mikheev, M.V., Sisler, J.R., Esposito, J.J., Jahrling, P.B., Moss, B., Sandakhchiev, L.S., 2001. Human monkeypox and smallpox viruses: genomic comparison. FEBS Lett. 509 (1), 66-70.

Tulman, E.R., Afonso, C.L., Lu, Z., Zsak, L., Kutish, G.F., Rock, D.L., 2001. Genome of lumpy skin disease virus. J. Virol. 75, 7122-7130.

Tulman, E.R., Afonso, C.L., Lu, Z., Zsak, L., Kutish, G.F., Rock, D.L., 2004. The genome of Canarypox virus. J. Virol. 78 (1), 353-366.

Tulman, E.R., Afonso, C.L., Lu, Z., Zsak, L., Sur, J.H., Sandybaev, N.T., Kerembekova, U.Z., Zaitsev, V.L., Kutish, G.F., Rock, D.L., 2002. The genomes of sheeppox and Goatpox viruses. J. Virol. 76 (12), 60546061.

Tulman, E.R., Delhon, G., Afonso, C.L., Lu, Z., Zsak, L., Sandybaev, N.T., Kerembekova, U.Z., Zaitsev, V.L., Kutish, G.F., Rock, D.L., 2006. Genome of Horsepox virus. J. Virol. 80 (18), 9244-9258.

Willer, D., McFadden, G., Evans, D.H., 1999. The complete genome sequence of Shope (rabbit) fibroma virus. Virology 264 (2), 319-343. 